\title{
Iodoamination of Alkenyl Sulfonamides by Potassium Iodide and Hydrogen Peroxide in Aqueous Medium
}

Sabrina Giofrè,${ }^{\mathrm{a}}$ Roberto Sala,${ }^{\mathrm{b}}$ Egle Maria Beccalli, ${ }^{\mathrm{a}}$ Leonardo Lo Presti, ${ }^{\mathrm{c}}$ and Gianluigi Broggini ${ }^{\mathrm{b} *}$

\begin{abstract}
A procedure for the iodoamination of unfunctionalized olefins tethered to a tosyl-protected $\mathrm{NH}$-group has been developed. The combined use of $\mathrm{KI}$ and $\mathrm{H}_{2} \mathrm{O}_{2}$ in aqueous medium was effective for the preparation of iodomethyl-substituted nitrogen-containing heterocycles. The selective exo-trig iodocyclization provided 1,2-bifunctional 5-, 6-, and 7-membered cyclic skeletons.
\end{abstract}

\section{Introduction}

The development of efficient synthetic protocols which allow the formation of more than one bond in a single step plays a central role in organic synthesis. Recently, haloamination of carbon-carbon double bond involving an intramolecular carbon-nitrogen bond formation received great attention as fruitful methodology for the synthesis of nitrogen-containing heterocycles. ${ }^{[1-3]}$ The usefulness of heterocycles containing a vicinal haloamine moiety as versatile synthetic intermediates ${ }^{[4-7]}$ or as potential medicinal agents ${ }^{[8,9]}$ is well established.

Among the alkene halocyclizations, iodoamination reactions represent a powerful tool for the preparation of heterocycles suitable for further introduction of functionalities. ${ }^{[10,11]}$ The formation of a carbon-iodine bond provides high added value to the process as proven by the multi-faceted conversions which can undergo. While the most efficient procedures for the access to vicinal chloro- and bromoamines are based on transition metal-catalyzed reactions, direct formation of vicinal iodoamines from aminoalkenes can be typically realized by initial activation of the carbon-carbon double bond with different sources of electrophile. Molecular iodine and $\mathrm{N}$-iodosuccinimide (NIS) have been widely used with amide-type substrates (Scheme $1 \mathrm{a})^{[12-15]}$ as well as with alkenyl imidates (Scheme 1b). ${ }^{[16-18]}$ Alternatively, $\mathrm{KI}$ in the presence of a hypervalent iodine derivatives (Scheme 1c) ${ }^{[19]}$ or a transition metal-catalyst (Scheme 1d) ${ }^{[20-21]}$ were proven to be effective for this goal. Also NaI, combined with $\mathrm{MnI}_{2}$ as catalyst, has been proven a useful source of iodine for iodoamination of unfunctionalized olefins (Scheme 1e). ${ }^{[22-23]}$ It is worth noting also the iodocyclization of alkenyl carbamates performed with $\mathrm{Bu}_{4} \mathrm{NI}$ as iodine source with $\mathrm{NaNO}_{2}$ as catalyst and molecular oxygen as oxidant. ${ }^{[24]}$ 
The importance of the development of a mild and easy synthesis of vicinal iodoamine moieties is also due to their role as core scaffolds in various structures of bioactive compounds. In particular, some antitumor, anti-infective, and anti-inflammatory agents feature iodomethyl substituted nitrogencontaining heterocycles (Figure 1). ${ }^{[25-26]}$

Previous work:

a) lodine and NIS mediated aminoiodination on amide-type substrates ${ }^{[12-15]}$

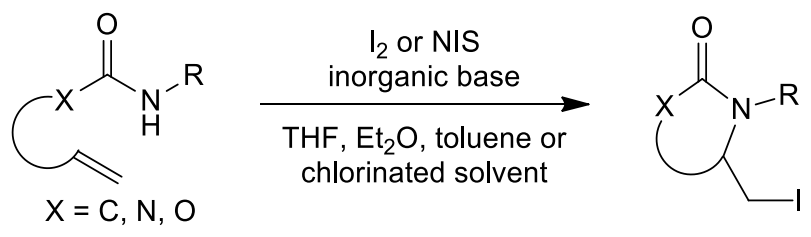

b) lodine and NIS mediated aminoiodination on imidate-type substrates ${ }^{[16-18]}$<smiles>[R]N=C([Y])[X]</smiles><smiles>OCCO[Mg]</smiles><smiles>[X]C1=NC(CI)CCC1[R]</smiles>

$\mathrm{X}=\mathrm{C}, \mathrm{O} ; \mathrm{Y}=\mathrm{CCl}_{3}, \mathrm{SMe} ; \mathrm{Z}=\mathrm{CCl}_{3}, \mathrm{OH}$

c) Hypervalent iodine promoted aminoiodination ${ }^{[19]}$

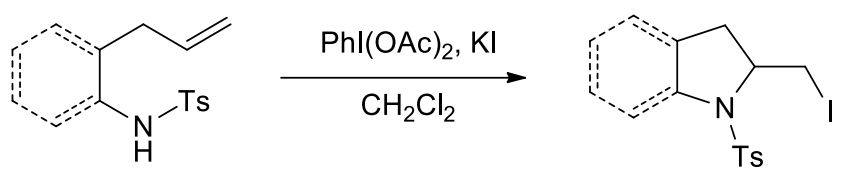

d) Transition metal-catalyzed aminoiodination ${ }^{[20,21]}$

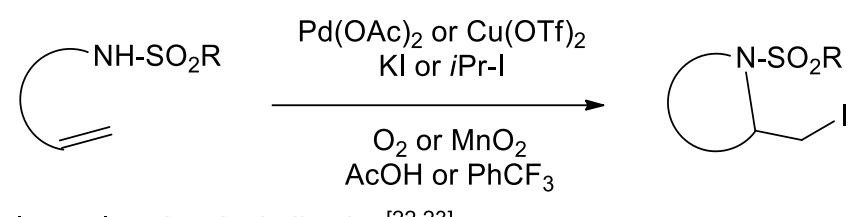

e) $\mathrm{Mnl}_{2}$-catalyzed aminoiodination ${ }^{[22,23]}$

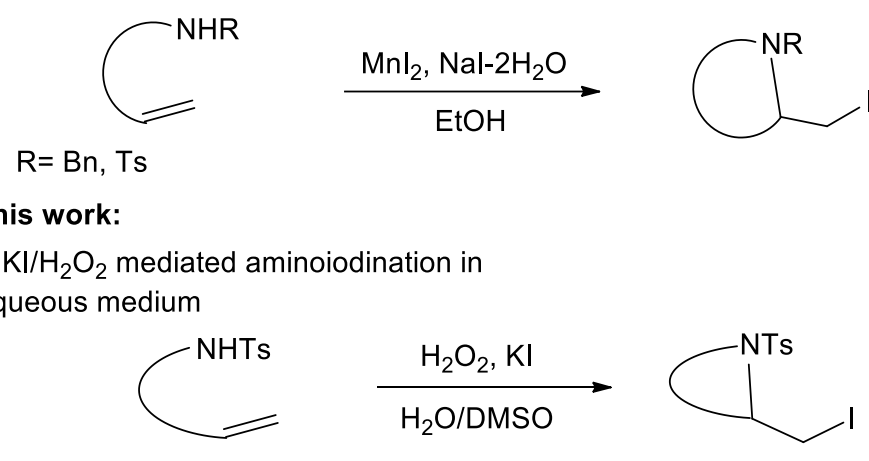

Scheme 1. Synthetic procedure for aminoiodination

Following our previous investigations on heterocyclization reactions in domino processes, ${ }^{[27-30]}$ we focused our attention on the search of alternative conditions for intramolecular amination reactions combined with iodine functionalization (Scheme 1f). Herein we report selected mild conditions relying 
on the use of $\mathrm{KI}$ as iodine source and $\mathrm{H}_{2} \mathrm{O}_{2}$ as oxidant agent which act in water at room temperature providing iodomethyl-substituted heterocycles. Although it is known that $\mathrm{H} 2 \mathrm{O} 2$ has been used combined to tetrabutylammonium iodide to promote an amination reaction, ${ }^{[31]}$ to the best of our knowledge the possibility to perform iodoamination reactions by the use of $\mathrm{KI} / \mathrm{H}_{2} \mathrm{O}_{2}$ is unknown in the literature.

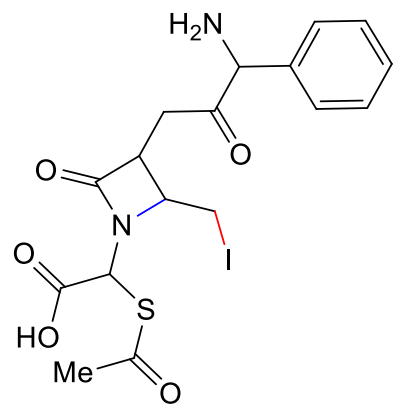

Anti-infective activities

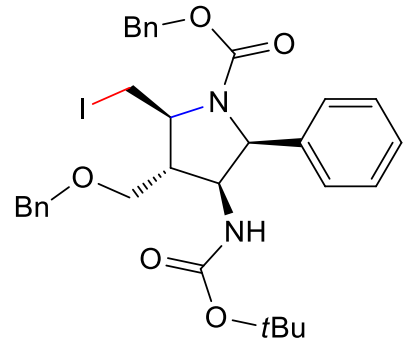

Anti-inflammatory agents

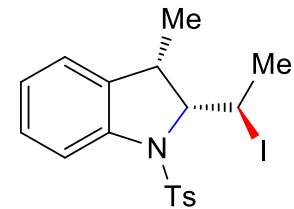

Antitumor activities

Antitumor activities

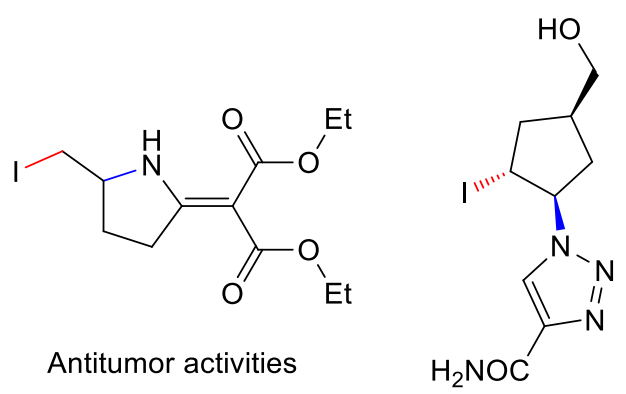<smiles></smiles>

Antitumor activities

Anti-infective activities

Figure 1. Selected bioactive vicinal iodoamine derivatives

\section{Results and Discussion}

$O$-Allyl- $N$-tosylcarbamate (1a) was investigated in preliminary experiments aimed to explore new conditions for the feasibility of the iodoamination reaction. Various combinations of iodine sources and oxidant agents in different solvents used to afford the oxazolidinone product $\mathbf{2 a}$ are collected in Table 1. A stoichiometric amount of molecular iodine or NIS in acetonitrile at room temperature furnished the iodoamination product in unsatisfactory yields in a complex crude mixture, in the latter case also due to the formation of the $O$-(2,3-diiodopropyl)- $N$-tosyl-carbamate (Table 1 , entries 1 and 2). The same acyclic diiodinated compound was obtained as by-product when the reaction was carried out in the presence of NIS and $\mathrm{CuCl}_{2}$ as the oxidant in oxygen atmosphere (Table 1, entry 3). A iodocyclizative process was observed when $\mathrm{I}_{2}$ and NIS were combined with PIFA, although the product resulted in the 3-unsubstituted 4-iodomethyloxazolidinone, isolated in both cases in moderate yields (Table 1, entries 4 and 5). Conversely, the use of PIFA was effective if used with KI as iodine source, providing 2a in $81 \%$ yield (Table 1 , entry 6 ). 
Other hypervalent iodine derivatives such as PIDA and $\mathrm{PhI}(\mathrm{mcba})_{2}$ were a good choice as promoter to afford 2a, although with a slight decrease in yield (Table 1, entries 7 and 8). Following these preliminary results, we focused on other conditions based on the presence of KI as iodine source. Using molecular iodine in $\mathrm{O}_{2}$ atmosphere combined with benzoquinone as further oxidant in acetonitrile, no formation of 2a was observed and carbamate 1a was completely recovered (Table 1, entry 9). Also $\mathrm{MnO}_{2}$ was tested as additive in the reaction mixture under oxygen atmosphere but $\mathbf{2 a}$ was achieved in low yield (Table 1, entry 10). Intriguingly, a consistent improvement of the outcome of the reaction was detected when $\mathrm{H}_{2} \mathrm{O}_{2}$ was used as the sole oxidant in the presence of $\mathrm{KI}$ and $\mathrm{CH}_{3} \mathrm{CN}$ as the solvent. These conditions determined a neat conversion of 1a, providing the desired iodoamination product in $79 \%$ yield (Table 1, entry 11). Use of DMF or dioxane led to a remarkable decrease of the yield (Table 1, entries 12 and 13), while water associated to $\mathrm{DMSO}$ or $\mathrm{CH}_{3} \mathrm{CN}$ as a co-solvent was gratifyingly proven to be a good medium to obtain the complete conversion of the substrate into $\mathbf{2 a}$ in mild conditions (Table 1, entries 14 and 15). Working in the conditions of Table 1, entry 14, the crude mixture resulted cleaner than the corresponding reaction carried out with $\mathrm{I}_{2}$ or NIS as iodine source. Besides, the product obtained might be collected through an easy filtration, due to its lower solubility in the $\mathrm{DMSO} / \mathrm{H}_{2} \mathrm{O}$ mixture compared to the starting material.

Table 1. Optimization of the reaction conditions.

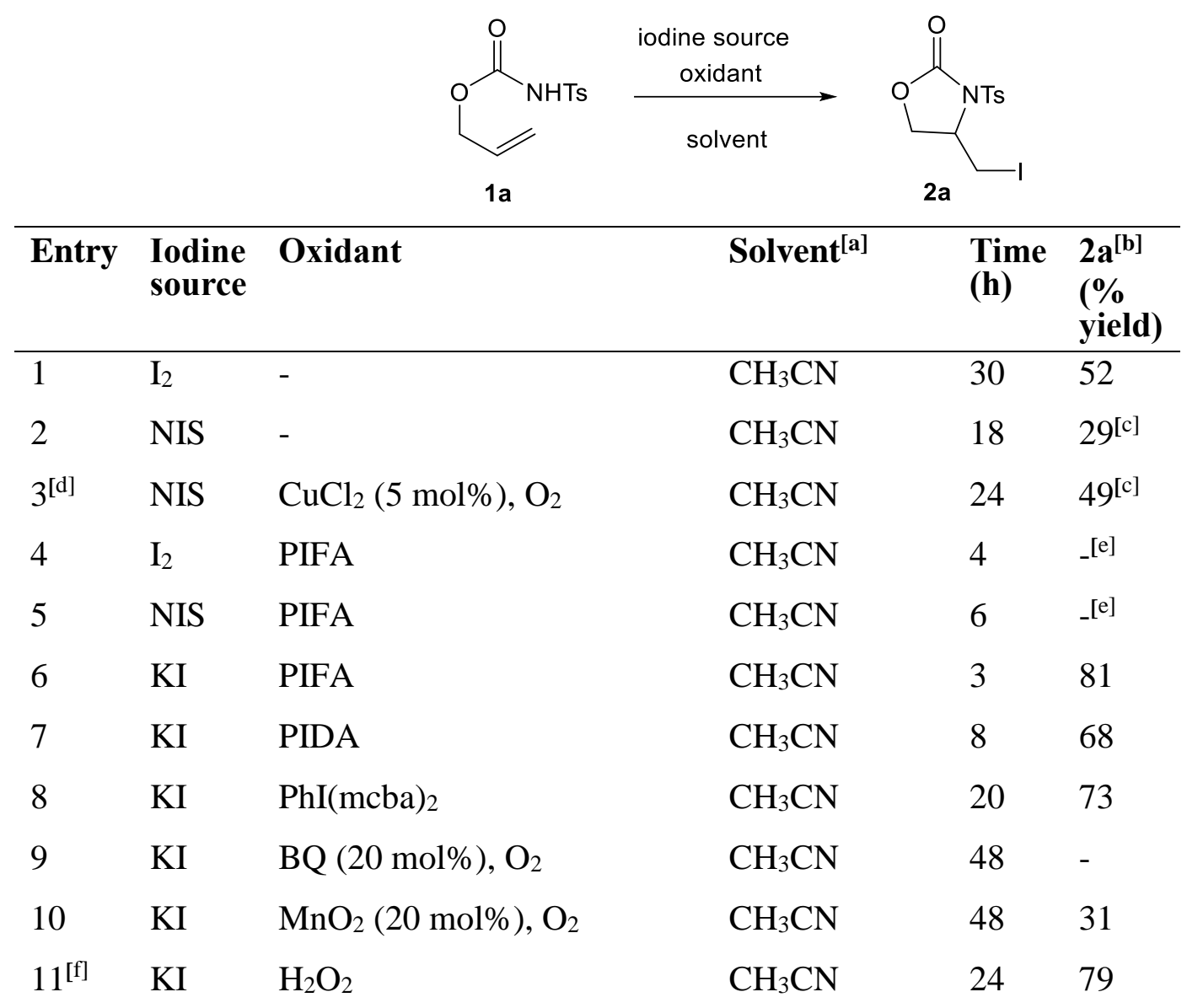




$\begin{array}{rlllll}12^{[\mathrm{f}]} & \mathrm{KI} & \mathrm{H}_{2} \mathrm{O}_{2} & \text { DMF } & 24 & 32 \\ 13^{[\mathrm{f}]} & \mathrm{KI} & \mathrm{H}_{2} \mathrm{O}_{2} & \text { Dioxane } & 24 & 54 \\ \mathbf{1 4}^{[\mathrm{f}]} & \mathbf{K I} & \mathbf{H}_{2} \mathrm{O}_{2} & \mathbf{H}_{2} \mathrm{O} / & \mathbf{2 4} & \mathbf{7 8} \\ & & & \mathbf{D M S O} & & \\ 15^{[\mathrm{f}]} & \mathrm{KI} & \mathrm{H}_{2} \mathrm{O}_{2} & \mathrm{H}_{2} \mathrm{O} / \mathrm{CH}_{3} \mathrm{CN}^{[\mathrm{h}]} & 24 & 67\end{array}$

${ }^{[\mathrm{a}]}$ The reactions were carried out at room temperature unless otherwise stated. ${ }^{[\mathrm{b}]}$ Yields of purified products. ${ }^{[\mathrm{c}]} \mathrm{O}$-(2,3-Diiodopropyl)- $N$-tosyl-carbamate has been isolated in $57 \%$ yield (entry 2) and $37 \%$ yield (entry 3 ). ${ }^{[\mathrm{d}]}$ The reaction was performed in $\mathrm{CH}_{3} \mathrm{CN}$ at reflux under $\mathrm{O} 2$ atmosphere. ${ }^{\text {e] }} 3$-Unsubstituted 4iodomethyl-oxazolidin-2-one has been isolated in 54\% yield (entry 4 ) and $43 \%$ yield (entry 5 ). ${ }^{[\mathrm{f}]}$ The reaction was carried out at room temperature using a $30 \%$ solution of $\mathrm{H}_{2} \mathrm{O}_{2}$ in water. ${ }^{[\mathrm{g}]}$ In 3:1 ratio. ${ }^{[\mathrm{h}]}$ In 2:1 ratio.

The result obtained with $\mathrm{KI} / \mathrm{H}_{2} \mathrm{O}_{2}$ in water as solvent at room temperature prompted us to explore the substrate scope of the iodoamination reaction in the conditions of Table 1, entry 19. Initially, variously substituted alkenyl carbamates were envisaged and the results of the iodocyclization reactions are reported in Table 2. The presence of substituents on the allylic moiety is compatible with reaction conditions, as demonstrated by the formation of compounds $\mathbf{2} \mathbf{b}-\mathbf{h}$, although a mild heating in some cases was required. Compounds $\mathbf{2 b}$ and $\mathbf{2 c}$ were obtained as a mixture of cis/trans diastereoisomers, easily separated and fully characterized.

The $\left(R^{*}, R^{*}\right)$-configuration of a representative iodo-derivative arising from $O$-allyl-carbamates mono-substituted at terminal position was confirmed by X-ray analysis of compound $\mathbf{2 f}$ (Figure 2), achieved by iodocyclization of the $O$-pent-2-enyl carbamate in the $(Z)$-configuration. The $O$-2cyclohexenyl- $N$-tosyl-carbamate furnished exclusively the bicyclic oxazolidinone $2 \mathbf{i}$ in $66 \%$ yield. The reaction conditions are also suitable for the iodoamination of $O$-alk-3-enyl carbamates, which provided the 1,3-oxazin-2-one products $\mathbf{2} \mathbf{j}$ and $\mathbf{2 k}$. 
Table 2. Reaction of iodoamination of alkenyl carbamates

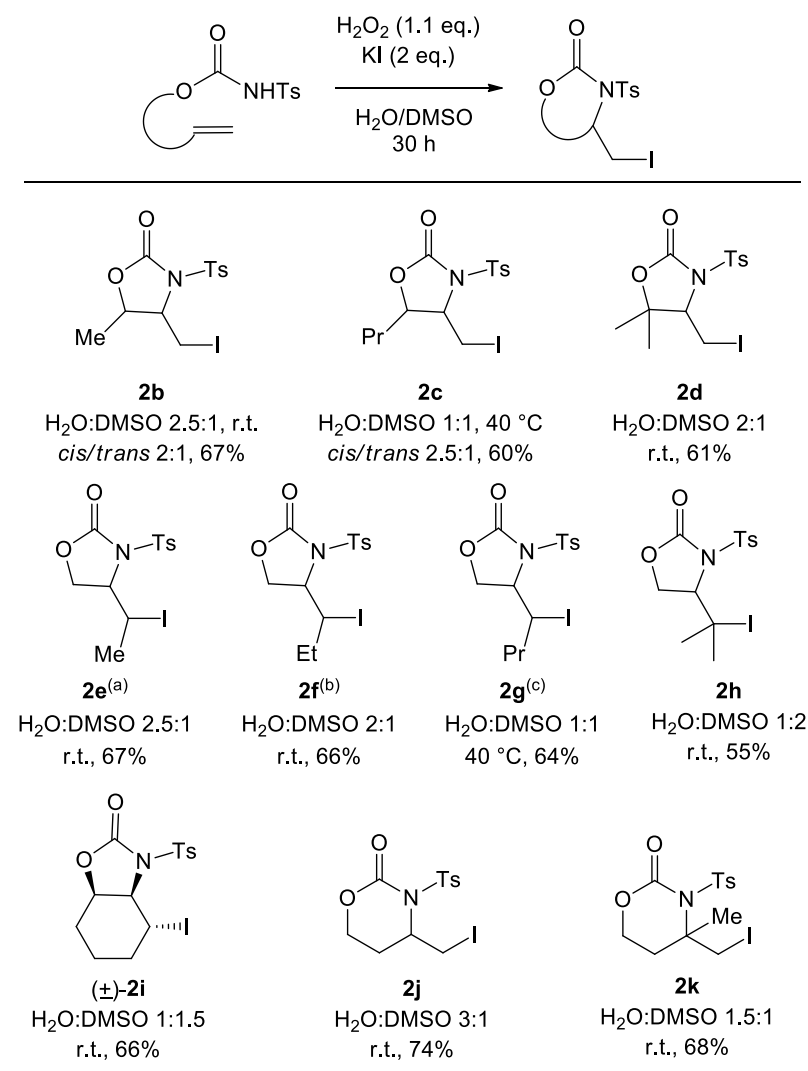

${ }^{[\mathrm{a}]}$ Starting from a mixture of $(E / Z)-\mathbf{1 e} .{ }^{[\mathrm{b}]}$ Starting from $(Z)-\mathbf{1 f} .{ }^{[\mathrm{c}]}$ Starting from $(E)-\mathbf{1 g}$.

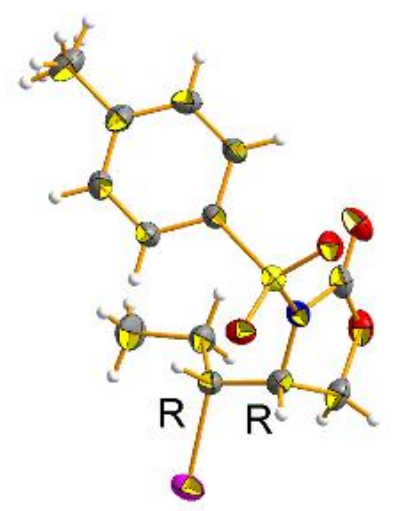

Figure 2. Molecular structure of the $(R, R)$ enantiomer of $\mathbf{2} \mathbf{f}$ at room temperature, as derived from single crystal X-ray diffraction. The methyl of the tosyl group is rotationally disordered with site occupation factors of 0.64(7) and 0.36(7). Thermal ellipsoids are drawn at the 30\% probability level. Configurational descriptors of the stereogenic centres are also shown.

Interestingly, the treatment of the optically active carbamate 3 with $\mathrm{H}_{2} \mathrm{O}_{2}$ and $\mathrm{KI}$ in $\mathrm{H}_{2} \mathrm{O} / \mathrm{DMSO}$ $1: 3$ at $40{ }^{\circ} \mathrm{C}$ resulted selectively in an exo-cyclization, providing the spiro-compound $\mathbf{4}$ as the sole product (Scheme 2). 
<smiles>C=C(C)C1CC=C(COC(=O)N[CH2+])CC1</smiles>

$(-)-3$

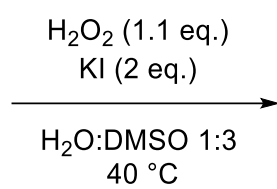

$40^{\circ} \mathrm{C}$

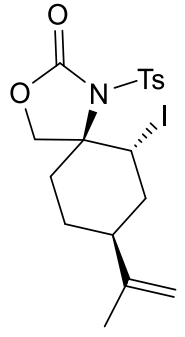

$(-)-4(63 \%)$

Scheme 2. Iodoamination of the optically active carbamate 3 .
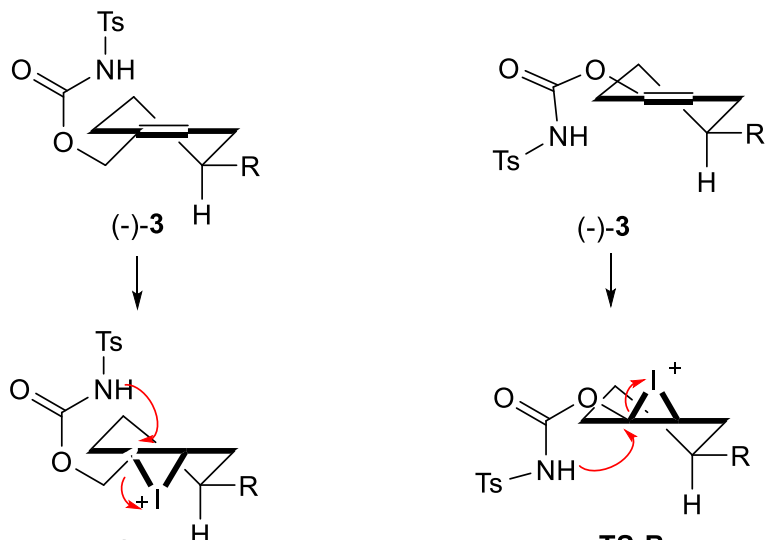

TS-A

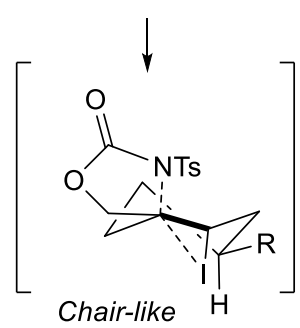

Transition State

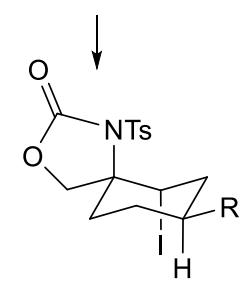

observed

$(-)-4$

(only diasteroisomer)

$\mathrm{R}=$ isopropenyl
$(-)-3$
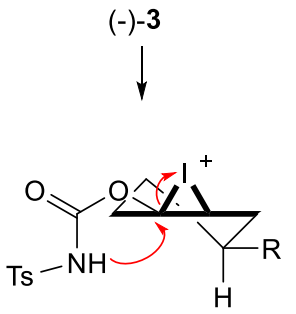

TS-B

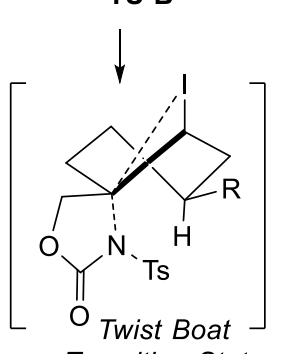

Transition State
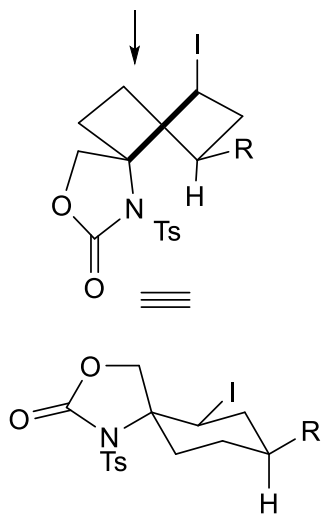

not observed

Scheme 3. Selective formation of the sole diastereoisomer (-)-4.

The stereoselective reaction path is due to the difference arisen from the torsional strain of the two plausible transition states having a substituent on the pseudo equatorial position (Scheme 3$).{ }^{[32-33]}$ The stability of the chair-like transition state (from TS-A) rather than the twist-boat state (from TS-B) afforded only the trans-diaxial addition product (-)-4. 
The configuration was unambiguously assigned by X-ray diffraction analysis (Figure 3).

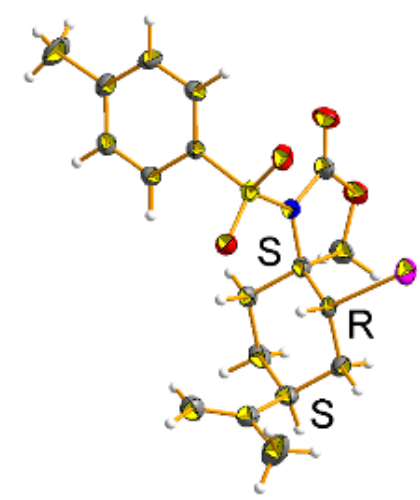

Figure 3. Molecular structure of 4 at room temperature, as derived from single crystal X-ray diffraction. Thermal ellipsoids are drawn at the $30 \%$ probability level. Configurational descriptors of the stereogenic centres are also shown.

Based on the observed results and literature data, ${ }^{[10,11]}$ a plausible mechanism for the iodocyclization is shown in Scheme 4 taking carbamate $1 \mathrm{a}$ as example. The $\mathrm{I}^{+}$species generated in situ from $\mathrm{KI}$ and $\mathrm{H}_{2} \mathrm{O}_{2}$ suggested the formation of the electrophilic iodinated intermediate $\mathbf{A},{ }^{[34-41]}$ from which the anti-attack by the nucleophilic tosylamino group afforded the 4-iodomethyl oxazolidinone product.

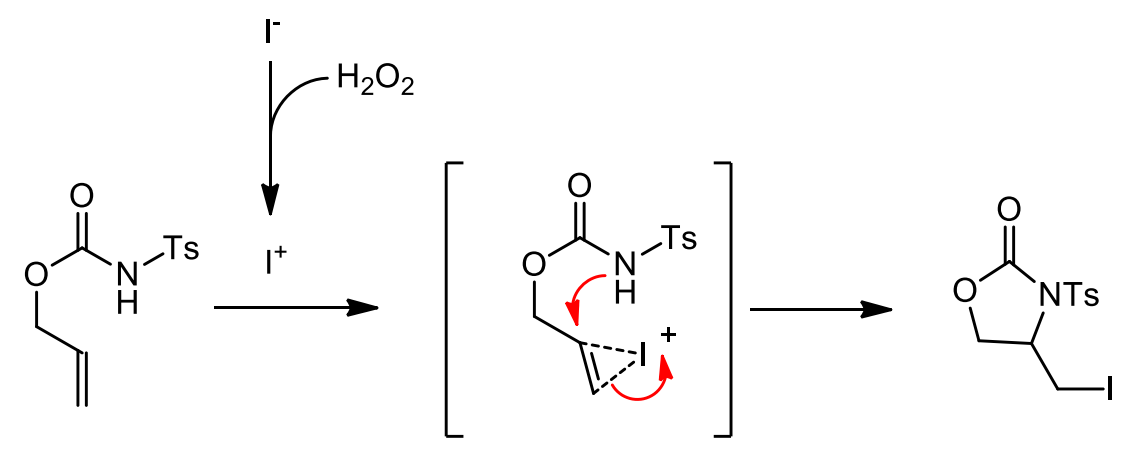

A

Scheme 4. Proposed iodoamination reaction mechanism

The use of $\mathrm{H}_{2} \mathrm{O}_{2}$ and $\mathrm{KI}$ for the iodocyclization of $N$-allyl- $N$ '-tosylureas resulted in a different outcome of the reaction depending on the substituent on the nitrogen atom. If the phenyl-substituted urea 5a provided the 4-iodomethyl-imidazolidinone $\mathbf{6 a}$ as the major product, the $N$-allyl- $N$-methyl-urea $\mathbf{5 b}$ followed mainly a iodoalkoxylation process giving the 2 -imino-oxazolidine $6 \mathbf{b}$ in $52 \%$ yield (Scheme 5). In both cases, minor products were detected in the crude mixtures, specifically arising from iodoalkoxylation (imino-oxazolidine 7a from 5a) and iodoamination (imidazolidinone $\mathbf{7 b}$ from $\mathbf{5 b}$ ) reactions. The possibility of $\mathrm{C}-\mathrm{O}$ vs $\mathrm{C}-\mathrm{N}$ bond formation in intramolecular reactions of secondary ureas is well known in literature. ${ }^{[42-44]}$ However, the low selectivity achieved in cyclization of compounds 
5a,b decreases the interest from the synthetic point of view for the application of this procedure to alkenyl ureas.
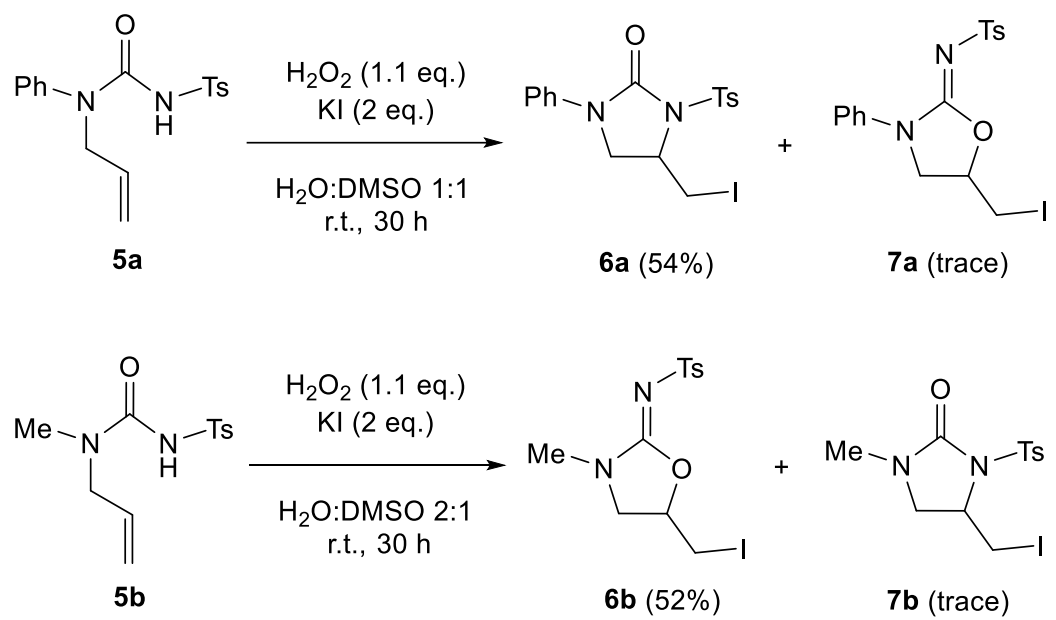

Scheme 5. Iodoamination reactions of allyl ureas

Further extension of the reaction scope was attempted taking into account other alkenyl sulphonamides.

The iodomethyl-substituted heterocyclic products, obtained by reaction with $\mathrm{KI}$ and $\mathrm{H}_{2} \mathrm{O}_{2}$ using $\mathrm{H}_{2} \mathrm{O} / \mathrm{DMSO}$ in a ratio depending on the solubility of the substrates, are collected in Table 3.

Table 3. Reaction of iodoamination of alkenyl tosylamines

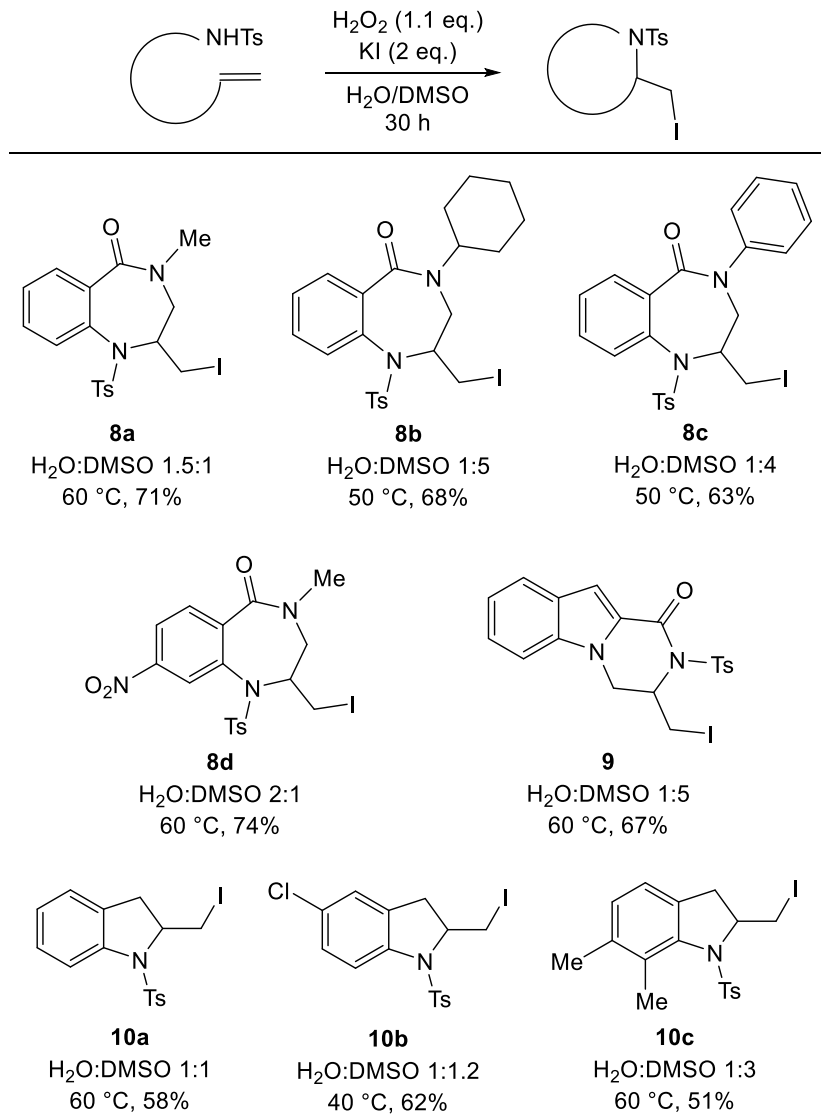


$N$-Allyl 2-tosylamino-benzamides, already used by our group for the synthesis of benzodiazepine scaffolds, ${ }^{[45]}$ were proven compatible with the iodoamination process, giving the 2-iodomethylsubstituted 1,2,3,4-tetrahydro-benzodiazepin-5-one derivatives 8a-d in 63-74\% yield. Satisfyingly, this procedure also represents a valuable alternative for the synthesis of these compounds, previously reported in the literature by displacement with KI of the first-formed chloromethyl derivative. ${ }^{[46]}$ The iodocyclization conditions were found effective also for the conversion of the 1-allyl-indole-2- $N$ tosylcarboxamide into the iodomethyl-substituted pyrazino[1,2-a]indole 9. It is noteworthy the reluctance of these substrates to afford haloamination processes in palladium-catalyzed reactions, ${ }^{[47]}$ as further proof of the fruitfulness of the $\mathrm{KI} / \mathrm{H}_{2} \mathrm{O}_{2}$ system. Finally, we explored the behaviour of 2-allyl$N$-tosylanilines, already successfully undergone to iodoamination in palladium-catalyzed conditions. ${ }^{[21]}$ Treatment of these substrates with the standard iodocyclization conditions provided the iodo-indolines 10a-c, although in moderate yields.

\section{Conclusions}

In summary, we have developed an efficient iodoamination procedure on alkenes bearing a secondary sulphonamide group based on the use of $\mathrm{KI} / \mathrm{H} 2 \mathrm{O} 2$ system. The current methodology allows to achieve easily different kind of iodomethyl-substituted nitrogen containing heterocycles by totally selective exocyclization which involves halogenation and the formation of an intramolecular $\mathrm{C}-\mathrm{N}$ bond. This iodoamination reaction proceeds smoothly in water needing the minimum amount of DMSO as cosolvent to solubilize the substrates.

\section{Experimental Section}

\section{Analytical Instruments}

Melting points were determined in a Stuart Scientific melting point apparatus in open capillary tubes and are uncorrected. Chemicals were obtained from Sigma Aldrich and used without further purification. HPLC analyses were carried out on a Kromasil 5-AmyCoat column $(4.6 \mathrm{~mm}$ i.d. $\times 250 \mathrm{~mm}, 5 \mu \mathrm{m}$, AkzoNobel). ESI mass spectra were recorded on a LCQ Advantage spectrometer from Thermo Finningan and a LCQ Fleet spectrometer from Thermo Scientific. The NMR spectroscopic experiments were carried out either on a Bruker AVANCE 400 (400 and $101 \mathrm{MHz}$ for ${ }^{1} \mathrm{H}$ and ${ }^{13} \mathrm{C}$, respectively), on a Varian OXFORD $300 \mathrm{MHz}$ (300 and $75 \mathrm{MHz}$ for ${ }^{1} \mathrm{H}$ and ${ }^{13} \mathrm{C}$, respectively), or on a Bruker Avance $300 \mathrm{MHz}$ spectrometers (300 and $75 \mathrm{MHz}$ for ${ }^{1} \mathrm{H}$ and ${ }^{13} \mathrm{C}$, respectively). Optical rotations were measured on a Perkin-Elmer 343 polarimeter at $20{ }^{\circ} \mathrm{C}$ (concentration in $\mathrm{g} / 100 \mathrm{~mL}$ ). Chemical shifts $\delta$ are given in ppm relative to the $\mathrm{CHCl}_{3}$ internal standard, and the coupling constants $J$ are reported in $\operatorname{Hertz}(\mathrm{Hz})$. 
General Procedure for the Aminoiodination Reaction. To a solution of the appropriate olefins (1 equiv.) in a mixture of DMSO and water (0.1M), potassium iodide (2 equiv.) was added at room temperature, followed by the addition of a solution of $30 \% \mathrm{H}_{2} \mathrm{O}_{2}$ (1.1 equiv) in water. The reaction was monitored by TLC. Then, the reaction mixture was quenched with $\mathrm{Na}_{2} \mathrm{~S}_{2} \mathrm{O}_{3}(1 \mathrm{M})$, and ethyl acetate was added. The organic layer was separated and washed with brine $(6 \times 15 \mathrm{~mL})$. Afterwards, the organic phase was dried over $\mathrm{Na}_{2} \mathrm{SO}_{4}$, filtered and evaporated under reduced pressure. The purification by flash silica gel column chromatography afforded the corresponding products.

4-(Iodomethyl)-3-tosyloxazolidin-2-one (2a). Compound 2a was prepared according to the general procedure $\left(\mathrm{H}_{2} \mathrm{O} / \mathrm{DMSO} 3: 1,30 \mathrm{~h}\right.$, room temperature) and isolated as white solid (yield $\left.78 \%\right)$ after flash column chromatography (hexane:EtOAc 3:1). ${ }^{1} \mathrm{H}$ NMR $\left(400 \mathrm{MHz}, \mathrm{CDCl}_{3}\right) \delta 7.90(\mathrm{~d}, J=8.2 \mathrm{~Hz}, 2 \mathrm{H})$, $7.30(\mathrm{~d}, J=8.1 \mathrm{~Hz}, 2 \mathrm{H}), 4.54-4.44(\mathrm{~m}, 1 \mathrm{H}), 4.36(\mathrm{t}, J=8.8 \mathrm{~Hz}, 1 \mathrm{H}), 4.09(\mathrm{dd}, J=9.1,3.9 \mathrm{~Hz}, 1 \mathrm{H}), 3.60$ $(\mathrm{dd}, J=10.3,2.3 \mathrm{~Hz}, 1 \mathrm{H}), 3.49-3.28(\mathrm{~m}, 1 \mathrm{H}), 2.39(\mathrm{~s}, 3 \mathrm{H})$. The data are in good agreement with those reported in the literature. ${ }^{[20]}$

Cis-4-(Iodomethyl)-5-methyl-3-tosyloxazolidin-2-one (cis-2b). Compound cis-2b was prepared according to the general procedure $\left(\mathrm{H}_{2} \mathrm{O} / \mathrm{DMSO} 2.5: 1,30 \mathrm{~h}\right.$, room temperature) and isolated as white solid (yield 45\%) after flash column chromatography (hexane:EtOAc 2:1). IR: 3428, 2975, 1778, 1359, $1163,1135,819,666,594,572,541 \mathrm{~cm}^{-1} .{ }^{1} \mathrm{H}$ NMR $\left(300 \mathrm{MHz}, \mathrm{CDCl}_{3}\right) \delta 8.00(\mathrm{~d}, J=8.4 \mathrm{~Hz}, 2 \mathrm{H}), 7.39$ $(\mathrm{d}, J=8.1 \mathrm{~Hz}, 2 \mathrm{H}), 4.91-4.79(\mathrm{~m}, 1 \mathrm{H}), 4.54(\mathrm{td}, J=7.2,2.2 \mathrm{~Hz}, 1 \mathrm{H}), 3.58-3.40(\mathrm{~m}, 2 \mathrm{H}), 2.48(\mathrm{~s}, 3 \mathrm{H})$, $1.61(\mathrm{~d}, J=6.7 \mathrm{~Hz}, 3 \mathrm{H}) .{ }^{13} \mathrm{C} \mathrm{NMR}\left(75 \mathrm{MHz}, \mathrm{CDCl}_{3}\right) \delta 151.2(\mathrm{~s}), 145.9$ (s), 134.7 (s), 129.9 (d), 128.7 (d), 75.0 (d), 59.3 (d), 21.8 (q), 13.5 (q), -0.5 (t). MS: (ESI) $m / z 418.05\left[\mathrm{M}+\mathrm{Na}^{+}\right]$. Anal. calcd for $\mathrm{C}_{12} \mathrm{H}_{14} \mathrm{NIO}_{4} \mathrm{~S}: \mathrm{C}, 36.47$; H, 3.57; N, 3.54. Found: C, 36.69; H, 3.32; N, 3.77.

Trans-4-(Iodomethyl)-5-methyl-3-tosyloxazolidin-2-one (trans-2b). Compound trans-2b was prepared according to the general procedure $\left(\mathrm{H}_{2} \mathrm{O} / \mathrm{DMSO} 2.5: 1,30 \mathrm{~h}\right.$, room temperature) and isolated as white solid (yield 22\%) after flash column chromatography (hexane:EtOAc 2:1). M.p. 146-147 ${ }^{\circ} \mathrm{C}$. IR: $3435,2984,1785,1595,1359,1166,11311088,816,665,596,568,539 \mathrm{~cm}^{-1} .{ }^{1} \mathrm{H}$ NMR (400 MHz, $\left.\mathrm{CDCl}_{3}\right) \delta 7.89(\mathrm{~d}, J=8.2 \mathrm{~Hz}, 2 \mathrm{H}), 7.30(\mathrm{~d}, J=8.1 \mathrm{~Hz}, 2 \mathrm{H}), 4.36(\mathrm{dd}, J=6.3,3.0 \mathrm{~Hz}, 1 \mathrm{H}), 4.02(\mathrm{dt}, J=$ 8.9, $2.7 \mathrm{~Hz}, 1 \mathrm{H}), 3.57(\mathrm{dd}, J=10.3,2.6 \mathrm{~Hz}, 1 \mathrm{H}), 3.31(\mathrm{t}, J=9.7 \mathrm{~Hz}, 1 \mathrm{H}), 2.39$ (s, 3H), $1.31(\mathrm{~d}, J=6.3$ $\mathrm{Hz}, 3 \mathrm{H}) .{ }^{13} \mathrm{C} \mathrm{NMR}\left(101 \mathrm{MHz}, \mathrm{CDCl}_{3}\right) \delta 148.0$ (s), 145.0 (s), 134.6 (s), 129.9 (d), 128.5 (d), 77.3 (d), 62.8 (d), 21.7 (q), 21.1 (q), 6.5 (t). MS: (ESI) m/z 418.12 [M+Na ${ }^{+}$. Anal. calcd for $\mathrm{C}_{12} \mathrm{H}_{14} \mathrm{NIO}_{4} \mathrm{~S}$ : C, 36.47; H, 3.57; N, 3.54. Found: C, 36.31; H, 3.80; N, 3.72. 
Cis-4-(Iodomethyl)-5-propyl-3-tosyloxazolidin-2-one (cis-2c). Compound cis-2c was prepared according to the general procedure $\left(\mathrm{H}_{2} \mathrm{O} / \mathrm{DMSO} 1: 1,24 \mathrm{~h}, 40{ }^{\circ} \mathrm{C}\right.$ ) and isolated as white solid (yield $28 \%$ ) after flash column chromatography (hexane:EtOAc 5:1 to 2:1). M.p.: 130-131 ${ }^{\circ}$ C. IR: $3435,2973,1788$, 1366, 1160, 657, $560 \mathrm{~cm}^{-1} .{ }^{1} \mathrm{H}$ NMR $\left(400 \mathrm{MHz}, \mathrm{CDCl}_{3}\right) \delta 8.01(\mathrm{~d}, J=8.3 \mathrm{~Hz}, 2 \mathrm{H}), 7.38(\mathrm{~d}, J=8.2 \mathrm{~Hz}$, 2H), 4.70-4.54 (m, 1H), 4.55-4.39 (m, 1H), 3.51-3.40 (m, 2H), 2.39 (s, 3H), 2.04-1.98 (m, 1H), 1.85$1.79(\mathrm{~m}, 1 \mathrm{H}), 1.80-1.46(\mathrm{~m}, 2 \mathrm{H}), 0.98(\mathrm{t}, J=7.4 \mathrm{~Hz}, 3 \mathrm{H}) .{ }^{13} \mathrm{C} \mathrm{NMR}\left(101 \mathrm{MHz}, \mathrm{CDCl}_{3}\right) \delta 151.4(\mathrm{~s})$, $145.0(\mathrm{~s}), 135.0$ (s), 130.0 (d), 128.9 (d), 78.8 (d), 59.1 (d), $29.6(\mathrm{t}), 21.9(\mathrm{q}), 19.3(\mathrm{t}), 13.8(\mathrm{q}),-0.0(\mathrm{t})$. MS: (ESI) $m / z 424.82\left[\mathrm{M}^{+}\right], 446.54\left[\mathrm{M}+\mathrm{Na}^{+}\right]$. Anal. calcd for $\mathrm{C}_{14} \mathrm{H}_{18} \mathrm{NIO}_{4} \mathrm{~S}: \mathrm{C}, 39.73 ; \mathrm{H}, 4.29 ; \mathrm{N}, 3.31$. Found: C, 39.65; H, 4.08; N, 3.59.

Trans-4-(Iodomethyl)-5-propyl-3-tosyloxazolidin-2-one (trans-2c). Compound trans-2c was prepared according to the general procedure $\left(\mathrm{H}_{2} \mathrm{O} / \mathrm{DMSO} 1: 1,24 \mathrm{~h}, 40{ }^{\circ} \mathrm{C}\right)$ and isolated as white solid (yield 32\%) after flash column chromatography hexane:EtOAc 5:1 to 2:1). M.p. $118-119{ }^{\circ} \mathrm{C}$. IR: 3437 , 2969, 1786, 1361, 1158, 655, $563 \mathrm{~cm}^{-1} .{ }^{1} \mathrm{H}$ NMR (300 MHz, $\left.\mathrm{CDCl}_{3}\right) \delta 7.97$ (d, J=8.4 Hz, 2H), 7.39 (d, $J=8.2 \mathrm{~Hz}, 2 \mathrm{H}), 4.39-4.24(\mathrm{~m}, 1 \mathrm{H}), 4.15(\mathrm{dt}, J=8.8,2.8 \mathrm{~Hz}, 1 \mathrm{H}), 3.64(\mathrm{dd}, J=10.3,2.8 \mathrm{~Hz}, 1 \mathrm{H}), 3.42$ $(\mathrm{dd}, J=10.2,8.8 \mathrm{~Hz}, 1 \mathrm{H}), 2.48(\mathrm{~s}, 3 \mathrm{H}), 1.74-1.27(\mathrm{~m}, 4 \mathrm{H}), 0.95(\mathrm{t}, J=7.3 \mathrm{~Hz}, 3 \mathrm{H}) .{ }^{13} \mathrm{C} \mathrm{NMR}(75 \mathrm{MHz}$, $\left.\mathrm{CDCl}_{3}\right) \delta 145.93(\mathrm{~s}), 134.6$ (s), 129.9 (d), 128.5 (d), 80.4 (d), 61.2 (d), 36.0 (t), 21.7 (q), 17.3 (d), 13.5 (q), 7.0 (t). MS: (ESI) $m / z 424.82\left[\mathrm{M}^{+}\right], 446.54\left[\mathrm{M}+\mathrm{Na}^{+}\right]$. Anal. calcd for $\mathrm{C}_{14} \mathrm{H}_{18} \mathrm{NIO}_{4} \mathrm{~S}: \mathrm{C}, 39.73 ; \mathrm{H}$, 4.29; N, 3.31. Found: C, 39.46; H, 4.55; N, 3.04.

4-(Iodomethyl)-5,5-dimethyl-3-tosyloxazolidin-2-one (2d). Compound $2 \mathrm{~d}$ was prepared according to the general procedure $\left(\mathrm{H}_{2} \mathrm{O} / \mathrm{DMSO} 2: 1,30 \mathrm{~h}\right.$, room temperature) and isolated as white solid (yield 56\%) after flash chromatography (hexane:EtOAc 3:1). ${ }^{1} \mathrm{H} \mathrm{NMR}\left(300 \mathrm{MHz}, \mathrm{CDCl}_{3}\right) \delta 7.98(\mathrm{~d}, J=8.3 \mathrm{~Hz}, 2 \mathrm{H})$, $7.37(\mathrm{~d}, J=8.1 \mathrm{~Hz}, 2 \mathrm{H}), 4.17(\mathrm{dd}, J=7.6,1.9 \mathrm{~Hz}, 1 \mathrm{H}), 3.59-3.41(\mathrm{~m}, 2 \mathrm{H}), 2.46(\mathrm{~s}, 3 \mathrm{H}), 1.61(\mathrm{~s}, 3 \mathrm{H})$, $1.39(\mathrm{~s}, 3 \mathrm{H})$. The data are in good agreement with those reported in the literature. ${ }^{[24]}$

4-(1-Iodopropyl)-3-tosyloxazolidin-2-one (2e). Compound 2e was prepared according to the general procedure $\left(\mathrm{H}_{2} \mathrm{O} / \mathrm{DMSO} 2.5: 1,30 \mathrm{~h}\right.$, room temperature) and isolated as white solid (yield 67\%) after flash column chromatography (hexane:EtOAc 2:1). M.p. 164-165 ${ }^{\circ}$ C. IR: 2998, 1781, 1602, 1346, 1174 , $11261075,810,603,575,549 \mathrm{~cm}^{-1} .{ }^{1} \mathrm{H}$ NMR $\left(400 \mathrm{MHz}, \mathrm{CDCl}_{3}\right) \delta 7.92(\mathrm{~d}, J=8.2 \mathrm{~Hz}, 2 \mathrm{H}), 7.30(\mathrm{~d}, J$ $=8.1 \mathrm{~Hz}, 2 \mathrm{H}), 4.81-4.62(\mathrm{~m}, 1 \mathrm{H}), 4.33(\mathrm{t}, J=9.0 \mathrm{~Hz}, 1 \mathrm{H}), 4.14(\mathrm{dd}, J=9.0,3.7 \mathrm{~Hz}, 1 \mathrm{H}), 3.90(\mathrm{dq}, J=$ 6.6, 3.1 Hz, 1H), $2.39(\mathrm{~s}, 3 \mathrm{H}), 1.77(\mathrm{~d}, J=7.2 \mathrm{~Hz}, 3 \mathrm{H}) .{ }^{13} \mathrm{C} \mathrm{NMR}\left(101 \mathrm{MHz}, \mathrm{CDCl}_{3}\right) \delta 151.6(\mathrm{~s}), 145.9$ (s), 134.6 (s), 129.8 (d), 128.8 (d), 66.4 (t), 61.2 (d), 29.2 (d), 23.1 (q), 21.7 (q). MS: (ESI) $\mathrm{m} / z 418.26$ $\left[\mathrm{M}+\mathrm{Na}^{+}\right]$. Anal. calcd for $\mathrm{C}_{12} \mathrm{H}_{14} \mathrm{NIO}_{4} \mathrm{~S}: \mathrm{C}, 36.47 ; \mathrm{H}, 3.57 ; \mathrm{N}, 3.54$. Found: $\mathrm{C}, 36.61 ; \mathrm{H}, 3.30 ; \mathrm{N}, 3.69$. 
4-(1-Iodopropyl)-3-tosyloxazolidin-2-one (2f). Compound $2 \mathrm{f}$ was prepared according to the general procedure $\left(\mathrm{H}_{2} \mathrm{O} / \mathrm{DMSO} 2: 1,30 \mathrm{~h}\right.$, room temperature) and isolated as white solid (yield 66\%) after flash column chromatography (hexane:EtOAc 1:1). M.p. 146-146 ${ }^{\circ} \mathrm{C}$. IR: 3435, 2971, 2873, 2850, 1784, 1369, 1356, 1175, 1164, 1116, 668, 596, $540 \mathrm{~cm}^{-1} .{ }^{1} \mathrm{H} \mathrm{NMR}\left(300 \mathrm{MHz}, \mathrm{CDCl}_{3}\right) \delta 7.97(\mathrm{~d}, J=7.9 \mathrm{~Hz}$, 2H), $7.40(\mathrm{~d}, J=7.9 \mathrm{~Hz}, 2 \mathrm{H}), 5.02-4.95(\mathrm{~m}, 1 \mathrm{H}), 4.60-4.50(\mathrm{~m}, 1 \mathrm{H}), 4.48-4.36(\mathrm{~m}, 2 \mathrm{H}), 2.48(\mathrm{~s}, 3 \mathrm{H})$, 1.62-1.45 (m, 2H), $1.01(\mathrm{t}, J=6.9 \mathrm{~Hz}, 3 \mathrm{H}) .{ }^{13} \mathrm{C} \mathrm{NMR}\left(75 \mathrm{MHz}, \mathrm{CDCl}_{3}\right) \delta 152.6(\mathrm{~s}), 146.5(\mathrm{~s}), 134.8$ (s), 130.3 (d), 128.7 (d), 66.8 (t), 61.0 (d), 37.5 (d), 24.1 (t), 22.1 (q), 14.6 (q). MS: (ESI) $\mathrm{m} / z 432.38$ $\left[\mathrm{M}+\mathrm{Na}^{+}\right]$. Anal. calcd for $\mathrm{C}_{13} \mathrm{H}_{16} \mathrm{NIO}_{4} \mathrm{~S}: \mathrm{C}, 38.15 ; \mathrm{H}, 3.94 ; \mathrm{N}, 3.42$. Found: $\mathrm{C}, 37.98 ; \mathrm{H}, 4.17 ; \mathrm{N}, 3.15$.

4-(1-Iodobutyl)-3-tosyloxazolidin-2-one (2g). Compound $2 \mathrm{~g}$ was prepared according to the general procedure $\left(\mathrm{H}_{2} \mathrm{O} / \mathrm{DMSO} 1: 1,24 \mathrm{~h}, 40{ }^{\circ} \mathrm{C}\right)$ and isolated as white solid (yield 64\%) after flash column chromatography (hexane:EtOAc 4:1). M.p. $136-137{ }^{\circ} \mathrm{C}$. IR: 3439, 2965, 1784, 1352, 1121, 645, 565 $\mathrm{cm}^{-1} .{ }^{1} \mathrm{H}$ NMR $\left(400 \mathrm{MHz}, \mathrm{CDCl}_{3}\right) \delta 7.92(\mathrm{~d}, J=8.2 \mathrm{~Hz}, 2 \mathrm{H}), 7.30(\mathrm{~d}, J=8.1 \mathrm{~Hz}, 2 \mathrm{H}), 4.73-4.56(\mathrm{~m}$, $1 \mathrm{H}), 4.32$ (t, $J=9.0 \mathrm{~Hz}, 1 \mathrm{H}), 4.16(\mathrm{dd}, J=9.0,3.8 \mathrm{~Hz}, 1 \mathrm{H}), 4.09-3.98$ (m, 1H), 2.39 (s, 3H), 1.73-1.28 (m, 4H), 0.91 (t, $J=7.1 \mathrm{~Hz}, 3 \mathrm{H}) .{ }^{13} \mathrm{C}$ NMR (101 MHz, $\left.\mathrm{CDCl}_{3}\right) \delta 151.6$ (s), 145.8 (s), $134.6(\mathrm{~s}), 129.7$ (d), 128.8 (d), 66.6 (t), 60.1 (d), 38.6 (d), 37.3 (t), 22.7 (t), 21.7 (q), 13.1 (q). MS: (ESI) m/z 446.21 $\left[\mathrm{M}+\mathrm{Na}^{+}\right]$. Anal. calcd for $\mathrm{C}_{14} \mathrm{H}_{18} \mathrm{NIO}_{4} \mathrm{~S}: \mathrm{C}, 39.73 ; \mathrm{H}, 4.29 ; \mathrm{N}, 3.31$. Found: C, 39.56; H, 4.47; N, 3.06 .

4-(2-Iodopropan-2-yl)-3-tosyloxazolidin-2-one (2h). Compound $2 \mathrm{~h}$ was prepared according to the general procedure $\left(\mathrm{H}_{2} \mathrm{O} / \mathrm{DMSO}\right.$ 1:2, 30h, room temperature) and isolated as white solid (yield 55\%) after flash chromatography (hexane:EtOAc 2.5:1). M.p. 156-160 ${ }^{\circ} \mathrm{C}$ (decomp.). IR: 3396, 2921, 1783 , 1165, 1160, 665, 554, $538 \mathrm{~cm}^{-1} .{ }^{1} \mathrm{H} \mathrm{NMR}\left(300 \mathrm{MHz}, \mathrm{CDCl}_{3}\right) \delta 7.95(\mathrm{~d}, J=8.4 \mathrm{~Hz}, 2 \mathrm{H}), 7.38(\mathrm{~d}, J=$ $8.3 \mathrm{~Hz}, 2 \mathrm{H}), 4.79$ (dd, $J=8.0,1.6 \mathrm{~Hz}, 1 \mathrm{H}), 4.63$ (dd, $J=9.9,1.6 \mathrm{~Hz}, 1 \mathrm{H}), 4.38$ (dd, $J=9.7,7.9 \mathrm{~Hz}$, $1 \mathrm{H}), 2.48$ (s, 3H), 2.16 (s, 3H), 1.89 (s, 3H). ${ }^{13} \mathrm{C} \mathrm{NMR}\left(75 \mathrm{MHz}, \mathrm{CDCl}_{3}\right) \delta 153.2,145.9,134.7,129.8$, 128.5, 69.8, 67.0, 48.9, 35.4, 30.0, 21.7. MS: (ESI) $m / z$ 432,24 [M+Na ${ }^{+}$. Anal. calcd for $\mathrm{C}_{13} \mathrm{H}_{16} \mathrm{NIO}_{4} \mathrm{~S}$ : C, 38.15; H, 3.94; N, 3.42. Found: C, 38.21; H, 3.72; N, 3.59.

4-Iodo-3-tosylhexahydrobenzo[d]oxazol-2(3H)-one (2i). Compound $2 \mathbf{i}$ was prepared according to the general procedure $\left(\mathrm{H}_{2} \mathrm{O} / \mathrm{DMSO} 1: 1.5,30 \mathrm{~h}\right.$, room temperature) and isolated as white solid (yield 66\%) after flash chromatography (hexane:EtOAc 3:1). M.p.: 171-172 ${ }^{\circ} \mathrm{C}$. IR: $3294,2940,1787,1175,1163$, 671, 558, $541 \mathrm{~cm}^{-1} .{ }^{1} \mathrm{H}$ NMR (400 MHz, $\left.\mathrm{CDCl}_{3}\right) \delta 7.98(\mathrm{~d}, J=8.2 \mathrm{~Hz}, 2 \mathrm{H}), 7.39$ (d, $\left.J=8.1 \mathrm{~Hz}, 2 \mathrm{H}\right)$, $5.00(\mathrm{dd}, J=8.0,3.9 \mathrm{~Hz}, 1 \mathrm{H}), 4.77(\mathrm{dd}, J=11.1,6.2 \mathrm{~Hz}, 1 \mathrm{H}), 4.67$ (dd, $J=6.5,3.6 \mathrm{~Hz}, 1 \mathrm{H}), 2.48$ (s, 3H), 2.28 - $1.58(\mathrm{~m}, 6 \mathrm{H}) .{ }^{13} \mathrm{C} \mathrm{NMR}\left(101 \mathrm{MHz}, \mathrm{CDCl}_{3}\right) \delta 145.9$ (s), 129.8 (d), 128.6 (d), $74.2(\mathrm{~d}), 63.3$ (d), 28.9 (t), 25.6 (d), $25.1(\mathrm{t}), 21.7$ (q), $18.1(\mathrm{t})$. MS: (ESI) $\mathrm{m} / \mathrm{z} 444.32\left[\mathrm{M}+\mathrm{Na}^{+}\right]$. Anal. calcd for $\mathrm{C}_{14} \mathrm{H}_{16} \mathrm{NIO}_{4} \mathrm{~S}: \mathrm{C}, 39.92 ; \mathrm{H}, 3.83$; N, 3.33. Found: C, 40.13; H, 3.55; N, 3.24. 
4-(Iodomethyl)-3-tosyl-1,3-oxazinan-2-one (2j). Compound $2 \mathbf{j}$ was prepared according to the general procedure $\left(\mathrm{H}_{2} \mathrm{O} / \mathrm{DMSO} 3: 1,30 \mathrm{~h}\right.$, room temperature) and isolated as white solid (yield $\left.74 \%\right)$ after flash chromatography (hexane:EtOAc 2:1). M.p.: $127-128^{\circ}$ C. IR: 3324, 1722, 1358, 1271, 1170, 1143, 669, 612, 542, $533 \mathrm{~cm}^{-1} .{ }^{1} \mathrm{H}$ NMR $\left(400 \mathrm{MHz}, \mathrm{CDCl}_{3}\right) \delta 7.84(\mathrm{~d}, J=8.2 \mathrm{~Hz}, 2 \mathrm{H}), 7.25(\mathrm{~d}, J=8.1 \mathrm{~Hz}, 2 \mathrm{H})$, 4.75-4.57 (m, 1H), 4.33-4.14 (m, 2H), 3.69 (dd, $J=10.1,3.0 \mathrm{~Hz}, 1 \mathrm{H}), 3.24(\mathrm{t}, J=10.6 \mathrm{~Hz}, 1 \mathrm{H}), 2.53-$ $2.42(\mathrm{~m}, 1 \mathrm{H}), 2.36(\mathrm{~s}, 3 \mathrm{H}), 2.22-2.06(\mathrm{~m}, 1 \mathrm{H}) .{ }^{13} \mathrm{C} \mathrm{NMR}\left(101 \mathrm{MHz}, \mathrm{CDCl}_{3}\right) \delta 148.0$ (s), $145.4(\mathrm{~s}), 135.2$ (s), 129.5 (d), 129.1 (d), 63.9 (t), 55.4 (d), 26.0 (t), 21.7 (q), 5.3 (t). MS: (ESI) m/z 418.51 [M+Na Anal. calcd for $\mathrm{C}_{12} \mathrm{H}_{14} \mathrm{NIO}_{4} \mathrm{~S}$ : C, 36.47; H, 3.57; N, 3.54. Found: C, 36.41; H, 3.75; N, 3.39.

4-(Iodomethyl)-4-methyl-3-tosyl-1,3-oxazinan-2-one (2k). Compound $2 \mathrm{k}$ was prepared according to the general procedure $\left(\mathrm{H}_{2} \mathrm{O} / \mathrm{DMSO} 1.5: 1,30 \mathrm{~h}\right.$, room temperature) and isolated as colorless oil (yield 68\%) after flash chromatography (hexane:EtOAc 4:1). IR: 2936, 1755, 1342, 1176, 667, 562, $546 \mathrm{~cm}^{-}$ ${ }^{1} .{ }^{1} \mathrm{H}$ NMR $\left(400 \mathrm{MHz}, \mathrm{CDCl}_{3}\right) \delta 7.92(\mathrm{~d}, J=8.1 \mathrm{~Hz}, 2 \mathrm{H}), 7.30(\mathrm{~d}, J=8.2 \mathrm{~Hz}, 2 \mathrm{H}), 4.30(\mathrm{~d}, J=10.4 \mathrm{~Hz}$, $1 \mathrm{H}), 4.23-4.15(\mathrm{~m}, 2 \mathrm{H}), 3.89(\mathrm{~d}, J=10.4 \mathrm{~Hz}, 1 \mathrm{H}), 2.57-2.45(\mathrm{~m}, 1 \mathrm{H}), 2.42(\mathrm{~s}, 3 \mathrm{H}), 2.11-1.96(\mathrm{~m}, 4 \mathrm{H})$. ${ }^{13} \mathrm{C} \mathrm{NMR}\left(101 \mathrm{MHz}, \mathrm{CDCl}_{3}\right) \delta 149.3,144.9,136.6,129.4,129.1,64.0,62.6,38.3,25.8,21.6,13.0 . \mathrm{MS}$ : (ESI) $m / z$ 432.45 [M+Na+]. Anal. calcd for $\mathrm{C}_{13} \mathrm{H}_{16} \mathrm{NIO}_{4} \mathrm{~S}$ : C, 38.15; H, 3.94; N, 3.42. Found: C, 37.99; H, 4.13; N, 3.53.

(5S,6R,8S)-6-Iodo-8-(prop-1-en-2-yl)-1-tosyl-3-oxa-1-azaspiro[4.5]decan-2-one (4). Compound 4 was prepared according to the general procedure $\left(\mathrm{H}_{2} \mathrm{O} / \mathrm{DMSO} 1: 3,24 \mathrm{~h}, 40{ }^{\circ} \mathrm{C}\right)$ and isolated as white solid (yield 63\%) after flash chromatography (hexane:EtOAc 3:1). M.p.: 160-164 ${ }^{\circ} \mathrm{C}$ (decomp.). IR: $3358,3261,2959,2922,1785,1188,1168,1084,667,562,545 \mathrm{~cm}^{-1} .{ }^{1} \mathrm{H} \mathrm{NMR}\left(300 \mathrm{MHz}, \mathrm{CDCl}_{3}\right) \delta$ $8.06(\mathrm{~d}, J=8.4 \mathrm{~Hz}, 2 \mathrm{H}), 7.35$ (d, $J=8.1 \mathrm{~Hz}, 2 \mathrm{H}), 5.46$ (dd, $J=13.6,4.1 \mathrm{~Hz}, 1 \mathrm{H}), 5.17(\mathrm{~s}, 1 \mathrm{H}), 5.01$ (s, $1 \mathrm{H}), 4.48(\mathrm{~d}, J=8.3 \mathrm{~Hz}, 1 \mathrm{H}), 4.31(\mathrm{~d}, J=8.7 \mathrm{~Hz}, 1 \mathrm{H}), 3.03(\mathrm{td}, J=13.8,3.2 \mathrm{~Hz}, 1 \mathrm{H}), 2.93-2.82(\mathrm{~m}$, 1H), $2.46(\mathrm{~s}, 3 \mathrm{H}), 2.33-1.99(\mathrm{~m}, 4 \mathrm{H}), 1.83(\mathrm{~s}, 3 \mathrm{H}), 1.71-1.56(\mathrm{~m}, 1 \mathrm{H}) .{ }^{13} \mathrm{C} \mathrm{NMR}\left(300 \mathrm{MHz}, \mathrm{CDCl}_{3}\right) \delta$ $151.0(\mathrm{~s}), 145.5(\mathrm{~s}), 143.1(\mathrm{~s}), 135.1(\mathrm{~s}), 129.7$ (d), 129.2 (d), $112.7(\mathrm{t}), 71.5(\mathrm{t}), 70.0(\mathrm{~s}), 40.5(\mathrm{~d}), 39.2$

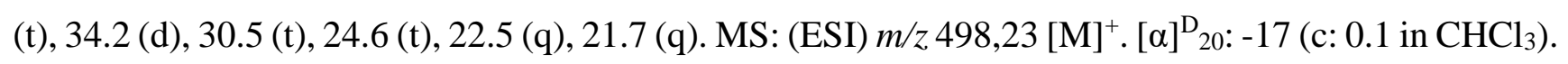
Anal. calcd for $\mathrm{C}_{18} \mathrm{H}_{22} \mathrm{NIO}_{4} \mathrm{~S}$ : C, 45.48; H, 4.67; N, 2.95. Found: C, 45.44; H, 4.89; N, 3.11 .

4-(Iodomethyl)-1-phenyl-3-tosylimidazolidin-2-one (6a). Compound 6a was prepared according to the general procedure $\left(\mathrm{H}_{2} \mathrm{O} / \mathrm{DMSO} 1: 1,30 \mathrm{~h}\right.$, room temperature) and isolated as pale yellow solid (yield $54 \%$ ) after flash chromatography (hexane:EtOAc $2.5: 1$ ). M.p. $148-149{ }^{\circ}$ C. IR: $3023,2958,1716,1599$, 1504, 1359, 1170, 1131, 751, 666, 592, $554 \mathrm{~cm}^{-1} .{ }^{1} \mathrm{H} \mathrm{NMR}\left(300 \mathrm{MHz}, \mathrm{CDCl}_{3}\right) \delta 8.02(\mathrm{~d}, J=8.4 \mathrm{~Hz}$, 2H), 7.53-7.43 (m, 2H), $7.35(\mathrm{t}, J=8.0 \mathrm{~Hz}, 4 \mathrm{H}), 7.14(\mathrm{t}, J=7.4 \mathrm{~Hz}, 1 \mathrm{H}), 4.54(\mathrm{tt}, J=8.8,3.2 \mathrm{~Hz}, 1 \mathrm{H})$, 
$4.09(\mathrm{t}, J=9.4 \mathrm{~Hz}, 1 \mathrm{H}), 3.78(\mathrm{dd}, J=10.2,2.7 \mathrm{~Hz}, 1 \mathrm{H}), 3.68(\mathrm{dd}, J=9.6,3.7 \mathrm{~Hz}, 1 \mathrm{H}), 3.54(\mathrm{dd}, J=$ 10.2, $8.4 \mathrm{~Hz}, 1 \mathrm{H}) .7 .54-7.44(\mathrm{~m}, 2 \mathrm{H}), 7.41-7.32(\mathrm{~m}, 4 \mathrm{H}), 7.14(\mathrm{t}, J=7.4 \mathrm{~Hz}, 1 \mathrm{H}), 4.72-4.41(\mathrm{~m}, 1 \mathrm{H})$, 4.09 (t, $J=9.4 \mathrm{~Hz}, 1 \mathrm{H}), 3.73(\mathrm{ddd}, J=29.8,9.9,3.2 \mathrm{~Hz}, 1 \mathrm{H}), 3.54(\mathrm{dd}, J=10.2,8.4 \mathrm{~Hz}, 1 \mathrm{H}), 2.46$ (s, 3H). ${ }^{13} \mathrm{C} \mathrm{NMR}\left(75 \mathrm{MHz}, \mathrm{CDCl}_{3}\right) \delta 151.1$ (s), 145.2 (s), 137.9 (s), 135.5 (s), 129.7 (d), 129.0 (d), 128.5 (d), 124.6 (d), 118.9 (d), 52.9 (d), 49.6 (t), 21.6 (q), 9.3 (t). MS: (ESI) $m / z, 479.20\left[\mathrm{M}+\mathrm{Na}^{+}\right]$. Anal. calcd for $\mathrm{C}_{17} \mathrm{H}_{17} \mathrm{~N}_{2} \mathrm{IO}_{3} \mathrm{~S}$ : C, 44.75; H, 3.76; N, 6.14. Found: C, 44.97; H, 3.50; N, 5.88.

\section{(Z)-N-(5-(Iodomethyl)-3-methyloxazolidin-2-ylidene)-4-methylbenzenesulfonamide}

(6b).

Compound 6b was prepared according to the general procedure $\left(\mathrm{H}_{2} \mathrm{O} / \mathrm{DMSO} 2: 1\right.$, 30h, room temperature) and isolated as white solid (yield 52\%) after flash chromatography (hexane:EtOAc 1:1). M.p. $120-121^{\circ} \mathrm{C}$. IR: 2920, 1728, 1358, 1172, 1140, 666, $590 \mathrm{~cm}^{-1} .{ }^{1} \mathrm{H}$ NMR $\left(300 \mathrm{MHz}, \mathrm{CDCl}_{3}\right) \delta 7.87$ $(\mathrm{d}, J=8.3 \mathrm{~Hz}, 2 \mathrm{H}), 7.25(\mathrm{~d}, J=8.1 \mathrm{~Hz}, 2 \mathrm{H}), 5.00-4.55(\mathrm{~m}, 1 \mathrm{H}), 3.79(\mathrm{t}, J=9.2 \mathrm{~Hz}, 1 \mathrm{H}), 3.37-3.22(\mathrm{~m}$, 3H), 2.96 (s, 3H), 2.41 (s, 3H). $\left.{ }^{13} \mathrm{C} \mathrm{NMR} \mathrm{(75} \mathrm{MHz,} \mathrm{CDCl}_{3}\right) \delta 157.6$ (s), 142.8 (s), 140.3 (s), 129.4 (d), 127.6 (d), 77.1 (d), 53.5 (t), 32.1 (q), 21.9 (q), 5.1 (t). MS: (ESI) $m / z 417.22\left[\mathrm{M}+\mathrm{Na}^{+}\right]$. Anal. calcd for $\mathrm{C}_{12} \mathrm{H}_{15} \mathrm{~N}_{2} \mathrm{IO}_{3} \mathrm{~S}$ : C, 36.56; H, 3.84; N, 7.11. Found: C, 36.29; H, 4.12; N, 6.91.

\section{2-(Iodomethyl)-4-methyl-1-tosyl-1,2,3,4-tetrahydro-5H-benzo[ $e][1,4]$ diazepin-5-one}

(8a).

Compound 8a was prepared according to the general procedure $\left(\mathrm{H}_{2} \mathrm{O} / \mathrm{DMSO} 1.5: 1,20 \mathrm{~h}, 60{ }^{\circ} \mathrm{C}\right)$ and isolated as white solid (yield 71\%) after flash chromatography (hexane:EtOAc 1.5:1). M.p. 156-156 ${ }^{\circ} \mathrm{C}$. IR: $3074,1670,1382,1157,1145,1049,712,667 \mathrm{~cm}^{-1} .{ }^{1} \mathrm{H}$ NMR $\left(300 \mathrm{MHz}, \mathrm{CDCl}_{3}\right) \delta 7.65(\mathrm{~d}, J=7.2$ $\mathrm{Hz}, 1 \mathrm{H}), 7.60-7.38$ (m, 5H), 7.27 (d, $J=7.7 \mathrm{~Hz}, 2 \mathrm{H}), 4.72-4.33(\mathrm{~m}, 1 \mathrm{H}), 3.75-3.45(\mathrm{~m}, 2 \mathrm{H}), 3.28-2.93$ $(\mathrm{m}, 2 \mathrm{H}), 2.62(\mathrm{~s}, 3 \mathrm{H}), 2.43(\mathrm{~s}, 3 \mathrm{H}) .{ }^{13} \mathrm{C} \mathrm{NMR}\left(75 \mathrm{MHz}, \mathrm{CDCl}_{3}\right) \delta 167.5(\mathrm{~s}), 144.1(\mathrm{~s}), 135.3(\mathrm{~s}), 134.4$ (s), 133.6 (d), 132.3 (s), 131.9 (d), 130.1 (d), 129.9 (d), 129.5 (d), 127.1 (d), 61.5 (d), 52.8 (t), 34.2 (q), 21.6 (q), 4.0 (t). MS: (ESI) $m / z, 471,22\left[\mathrm{M}+\mathrm{Na}^{+}\right]$. Anal. calcd for $\mathrm{C}_{18} \mathrm{H}_{19} \mathrm{~N}_{2} \mathrm{IO}_{3} \mathrm{~S}: \mathrm{C}, 45.97 ; \mathrm{H}, 4.07 ; \mathrm{N}$, 5.96. Found: C, 45.94; H, 4.23; N, 6.19 .

\section{4-Cyclohexyl-2-(iodomethyl)-1-tosyl-1,2,3,4-tetrahydro-5H-benzo $[e][1,4]$ diazepin-5-one}

(8b). Compound $\mathbf{8 b}$ was prepared according to the general procedure $\left(\mathrm{H}_{2} \mathrm{O} / \mathrm{DMSO} 1: 5,30 \mathrm{~h}, 50{ }^{\circ} \mathrm{C}\right)$ and isolated as white solid (yield 68\%) after flash chromatography (hexane:EtOAc 7:3). M.p. $=158-159{ }^{\circ} \mathrm{C}$. $\mathrm{IR}=2924,1640,1347,1157,1139,1055,710,662 \mathrm{~cm}^{-1} .{ }^{1} \mathrm{H}$ NMR $\left(400 \mathrm{MHz}, \mathrm{CDCl}_{3}\right): \delta 7.61(\mathrm{~d}, J=8$ $\mathrm{Hz}, 1 \mathrm{H}), 7.47-7.38(\mathrm{~m}, 5 \mathrm{H}), 7.17(\mathrm{~d}, J=8 \mathrm{~Hz}, 2 \mathrm{H}), 4.16-4.09(\mathrm{~m}, 1 \mathrm{H}), 3.84-3.78(\mathrm{~m}, 1 \mathrm{H}), 3.69$ (dd, $J=$ $15.5 \mathrm{~Hz}, 4.5 \mathrm{~Hz}, 1 \mathrm{H}), 3.53(\mathrm{dd}, J=9.9 \mathrm{~Hz}, 3.7 \mathrm{~Hz}, 1 \mathrm{H}), 2.97$ (t, $J=10.1 \mathrm{~Hz}, 1 \mathrm{H}), 2.73(\mathrm{dd}, J=15.4$, $11.9 \mathrm{~Hz}, 1 \mathrm{H}), 2.34$ (s, 3H), 1.74-0.81 (m, 10H). $\left.{ }^{13} \mathrm{C} \mathrm{NMR} \mathrm{(101} \mathrm{MHz,} \mathrm{CDCl}_{3}\right): \delta 167.5$ (s), $144.1(\mathrm{~s})$, 135.7 (s), 135.1 (s), 132.9 (d), 132.3 (s), 131.6 (d), 130.5 (d), 129.7 (d), 129.2 (d), 127.7 (d), 64.0 (d), 
$53.0(\mathrm{~d}), 45.6(\mathrm{t}), 30.4(\mathrm{t}), 30.1(\mathrm{t}), 25.7(\mathrm{t}), 25.3(\mathrm{t}), 25.3(\mathrm{t}), 21.5(\mathrm{q}), 4.0(\mathrm{t})$. MS: (ESI) $\mathrm{m} / \mathrm{z} 561.45$ $\left[\mathrm{M}+\mathrm{Na}^{+}\right]$. Anal. calcd for $\mathrm{C}_{23} \mathrm{H}_{27} \mathrm{~N}_{2} \mathrm{IO}_{3} \mathrm{~S}: \mathrm{C}, 51.30 ; \mathrm{H}, 5.05 ; \mathrm{N}, 5.20$. Found: C, 51.07; H, 5.28; N, 4.97.

2-(Iodomethyl)-4-phenyl-1-tosyl-1,2,3,4-tetrahydro-5H-benzo[e][1,4]diazepin-5-one

(8c).

Compound 8c was prepared according to the general procedure $\left(\mathrm{H}_{2} \mathrm{O} / \mathrm{DMSO} 1: 5,30 \mathrm{~h}, 50{ }^{\circ} \mathrm{C}\right)$ and isolated as white solid (yield 63\%) after flash chromatography (hexane:EtOAc 4:1). M.p.: 87-88 ${ }^{\circ} \mathrm{C}$. IR $=1653,1398,1350,1170,1087,1050,743,717 .{ }^{1} \mathrm{H}$ NMR $\left(400 \mathrm{MHz}, \mathrm{CDCl}_{3}\right): \delta 7.68(\mathrm{~d}, J=7.4 \mathrm{~Hz}$, 1H), 7.55-7.42 (m, 5H), 7.21-7.14 (m, 5H), 6.59 (d, $J=7.5 \mathrm{~Hz}, 2 \mathrm{H}), 4.46-4.40(\mathrm{~m}, 1 \mathrm{H}), 4.02(\mathrm{dd}, J=$ 15.1, 4.3Hz, 1H), 3.60-3.35 (m, 2H), $3.11(\mathrm{t}, J=9.8 \mathrm{~Hz}, 1 \mathrm{H}), 2.33(\mathrm{~s}, 3 \mathrm{H}) .{ }^{13} \mathrm{C} \mathrm{NMR}\left(101 \mathrm{MHz}, \mathrm{CDCl}_{3}\right)$ : $\delta 157.3$ (s), 144.3 (s), $141.4(\mathrm{~s}), 135.9$ (s), 134.8 (s), 133.4 (d), 132.5 (s), 132.0 (d), 130.5 (d), 130.0 (d), 129.4 (d), 128.0 (d), 127.6 (d), 126.8 (d), 125.3 (d), 62.0 (d), 54.3 (t), 21.5 (q), 4.2 (t). MS: (ESI) m/z $555.23\left[\mathrm{M}+\mathrm{Na}^{+}\right]$. Anal. calcd for $\mathrm{C}_{23} \mathrm{H}_{21} \mathrm{~N}_{2} \mathrm{IO}_{3} \mathrm{~S}: \mathrm{C}, 51.89 ; \mathrm{H}, 3.98 ; \mathrm{N}, 5.26$. Found: C, 52.01; H, 3.81; $\mathrm{N}, 5.45$.

\section{2-(Iodomethyl)-4-methyl-8-nitro-1-tosyl-1,2,3,4-tetrahydro-5H-benzo[e][1,4]diazepin-5-one (8d).} Compound 8d was prepared according to the general procedure $\left(\mathrm{H}_{2} \mathrm{O} / \mathrm{DMSO} 2: 1,24 \mathrm{~h}, 60{ }^{\circ} \mathrm{C}\right)$ and isolated as pale yellow solid (yield 74\%) after flash chromatography (hexane:EtOAc 1:1). M.p. 85$86{ }^{\circ} \mathrm{C}$. IR: $2921,1654,1334,1155,1111,709,666,583,571 \mathrm{~cm}^{-1} .{ }^{1} \mathrm{H}$ NMR $\left(300 \mathrm{MHz}, \mathrm{CDCl}_{3}\right) \delta 8.42$ $(\mathrm{d}, J=2.1 \mathrm{~Hz}, 1 \mathrm{H}), 8.30(\mathrm{dd}, J=8.5,2.2 \mathrm{~Hz}, 1 \mathrm{H}), 7.81(\mathrm{~d}, J=8.5 \mathrm{~Hz}, 1 \mathrm{H}), 7.42(\mathrm{~d}, J=8.3 \mathrm{~Hz}, 2 \mathrm{H})$, 7.32-7.22 (m, 2H), 4.66-4.28 (m, 1H), 3.66-3.48 (m, 2H), 3.28-3.07 (m, 2H), $2.64(\mathrm{~s}, 3 \mathrm{H}), 2.43(\mathrm{~s}, 3 \mathrm{H})$. ${ }^{13} \mathrm{C} \mathrm{NMR}\left(75 \mathrm{MHz}, \mathrm{CDCl}_{3}\right) \delta 144.8$ (s), 140.1 (s), 134.7 (s), 133.8 (s), 131.2 (d), 130.1 (d), 129.0 (d), 127.1 (d), 123.9 (d), 61.3 (d), 52.8 (t), 34.3 (q), 21.6 (q), 4.1 (t). MS: (ESI) $\mathrm{m} / z$ 538.43[M+Na ${ }^{+}$. Anal. calcd for $\mathrm{C}_{18} \mathrm{H}_{18} \mathrm{~N}_{3} \mathrm{IO}_{5} \mathrm{~S}: \mathrm{C}, 41.95 ; \mathrm{H}, 3.52 ; \mathrm{N}, 8.15$. Found: $\mathrm{C}, 42.08 ; \mathrm{H}, 3.43 ; \mathrm{N}, 7.96$.

3-(Iodomethyl)-2-tosyl-3,4-dihydropyrazino[1,2-a]indol-1(2H)-one (9). Compound 9 was prepared according to the general procedure $\left(\mathrm{H}_{2} \mathrm{O} / \mathrm{DMSO}\right.$ 1:5, 6h, room temperature) and isolated as white solid (yield 67\%) after flash chromatography (hexane:EtOAc 4:1). M.p. 173-174 ${ }^{\circ}$ C. IR: 3435, 2922, 1690, 1536, 1346, 1163, 742, 715, $556 \mathrm{~cm}^{-1} .{ }^{1} \mathrm{H}$ NMR (300 MHz, $\left.\mathrm{CDCl}_{3}\right) \delta 8.06(\mathrm{~d}, J=8.4 \mathrm{~Hz}, 2 \mathrm{H}), 7.71(\mathrm{~d}$, $J=8.2 \mathrm{~Hz}, 1 \mathrm{H}), 7.46-7.33(\mathrm{~m}, 5 \mathrm{H}), 7.26-7.13(\mathrm{~m}, 1 \mathrm{H}), 5.37-5.25(\mathrm{~m}, 1 \mathrm{H}), 5.13(\mathrm{~d}, J=13.2 \mathrm{~Hz}, 1 \mathrm{H})$, $4.33(\mathrm{dd}, J=12.8,3.0 \mathrm{~Hz}, 1 \mathrm{H}), 3.62-3.49(\mathrm{~m}, 1 \mathrm{H}), 3.17(\mathrm{dd}, J=11.6,10.3 \mathrm{~Hz}, 1 \mathrm{H}), 2.46(\mathrm{~s}, 3 \mathrm{H}) .{ }^{13} \mathrm{C}$ NMR (75 MHz, $\left.\mathrm{CDCl}_{3}\right) \delta 156.3(\mathrm{~s}), 145.5$ (s), 137.3 (s), 135.8 (s), 129.6 (d), 129.0 (d), 127.3 (s), 126.4 (d), 126.1 (s), 123.2 (d), 121.6 (d), 110.0 (d), 56.9 (d), 42.8 (t), 21.7 (q), 2.8 (t). MS: (ESI) $\mathrm{m} / \mathrm{z} 481.25$ $\left[\mathrm{M}^{+}\right], 503.38\left[\mathrm{M}+\mathrm{Na}^{+}\right]$. Anal. calcd for $\mathrm{C}_{19} \mathrm{H}_{17} \mathrm{~N}_{2} \mathrm{IO}_{3} \mathrm{~S}: \mathrm{C}, 47.51 ; \mathrm{H}, 3.57 ; \mathrm{N}, 5.83$. Found: C, 47.59; $\mathrm{H}$, $3.80 ; \mathrm{N}, 5.62$. 
2-(Iodomethyl)-1-tosylindoline (10a). Compound 10a was prepared according to the general procedure $\left(\mathrm{H}_{2} \mathrm{O} / \mathrm{DMSO}\right.$ 1:1, 24h, $\left.60^{\circ} \mathrm{C}\right)$ and isolated as pale yellow solid (yield 58\%) after flash chromatography (hexane:EtOAc 10:1). ${ }^{1} \mathrm{H}$ NMR (400 MHz, $\left.\mathrm{CDCl}_{3}\right) \delta=7.57(\mathrm{~d}, J=8.1 \mathrm{~Hz}, 1 \mathrm{H}), 7.48$ $(\mathrm{d}, J=8.1 \mathrm{~Hz}, 2 \mathrm{H}), 7.20-7.07$ (m, 3H), 7.00-6.93 (m, 2H), 4.30-4.24 (m, 1H), 3.58 (dd, $J=9.7,3.4 \mathrm{~Hz}$, $1 \mathrm{H}), 3.18(\mathrm{t}, J=9.9 \mathrm{~Hz}, 1 \mathrm{H}), 2.86(\mathrm{dd}, J=16.7,9.3 \mathrm{~Hz}, 1 \mathrm{H}), 2.76(\mathrm{dd}, J=16.7,3.0 \mathrm{~Hz}, 1 \mathrm{H}), 2.27$ (s, $3 \mathrm{H})$. The data are in good agreement with those reported in the literature. ${ }^{[18]}$

5-Chloro-2-(iodomethyl)-1-tosylindoline (10b). Compound 10b was prepared according to the general procedure $\left(\mathrm{H}_{2} \mathrm{O} / \mathrm{DMSO} 1: 1.2,20 \mathrm{~h}, 40{ }^{\circ} \mathrm{C}\right)$ and isolated as white solid (yield 62\%) after flash chromatography (hexane:EtOAc 10:1). ${ }^{1} \mathrm{H}$ NMR (300 $\left.\mathrm{MHz}, \mathrm{CDCl}_{3}\right) \delta=7.58-7.53(\mathrm{~m}, 3 \mathrm{H}), 7.23-7.16$ $(\mathrm{m}, 3 \mathrm{H}), 7.03(\mathrm{~s}, 1 \mathrm{H}), 4.36-4.30(\mathrm{~m}, 1 \mathrm{H}), 3.63(\mathrm{dd}, J=9.7,3.3 \mathrm{~Hz}, 1 \mathrm{H}), 3.26(\mathrm{t}, J=9.9 \mathrm{~Hz}, 1 \mathrm{H}), 2.89$ $2.82(\mathrm{~m}, 2 \mathrm{H}), 2.37(\mathrm{~s}, 3 \mathrm{H})$. The data are in good agreement with those reported in the literature. ${ }^{[18]}$

2-(Iodomethyl)-6,7-dimethyl-1-tosylindoline (10c). Compound 10c was prepared according to the general procedure $\left(\mathrm{H}_{2} \mathrm{O} / \mathrm{DMSO} 1: 2.5,20 \mathrm{~h}, 60^{\circ} \mathrm{C}\right)$ and isolated as pale yellow solid (yield $\left.51 \%\right)$ after flash chromatography (hexane:EtOAc 12:1). ${ }^{1} \mathrm{H}$ NMR $\left(300 \mathrm{MHz}, \mathrm{CDCl}_{3}\right) \delta 7.31(\mathrm{~d}, J=8.3 \mathrm{~Hz}, 2 \mathrm{H})$, $7.15(\mathrm{~d}, J=8.0 \mathrm{~Hz}, 2 \mathrm{H}), 6.96(\mathrm{t}, J=6.9 \mathrm{~Hz}, 2 \mathrm{H}), 6.77(\mathrm{~d}, J=7.5 \mathrm{~Hz}, 1 \mathrm{H}), 4.48-4.37(\mathrm{~m}, 1 \mathrm{H}), 3.36(\mathrm{dd}$, $J=9.8,5.1 \mathrm{~Hz}, 1 \mathrm{H}), 2.98(\mathrm{t}, J=9.9 \mathrm{~Hz}, 1 \mathrm{H}), 2.45(\mathrm{~s}, 3 \mathrm{H}), 2.38(\mathrm{~s}, 3 \mathrm{H}), 2.31(\mathrm{~s}, 3 \mathrm{H}), 2.12-1.98(\mathrm{~m}, 2 \mathrm{H})$. The data are in good agreement with those reported in the literature. ${ }^{[20]}$

General procedure for the X-ray diffraction analysis. Single-crystal X-ray diffraction experiments were carried out on a Bruker AXS three-circle diffractometer equipped with an Apex II CCD area detector. Data were collected using graphite-monochromated Mo K $\alpha$ radiation $(\lambda=0.71073 \AA)$ at a nominal Xrays power of $50 \mathrm{kV}$ x $30 \mathrm{~mA}$. A $100 \%$ complete full sphere of reflections was recorded up to a maximum resolution of $0.77 \AA$, resulting in 3578 (compound 2f) and 4460 (ompound 4) independent structure factor amplitudes. The latter were reduced with the SAINT+ software ${ }^{[48]}$ and corrected for absorption using the empirical procedure implemented in SADABS. ${ }^{[49]}$ The structures were solved by either iterative charge-flipping methods implemented in SUPERFLIP ${ }^{[50]}$ (compound 2f) or by direct methods (SIR92). ${ }^{[51]}$ Structure refinements were carried out in the independent atom approximation with the least squares algorithm implemented in shelxl. ${ }^{[52]}$ Molecular drawings were plotted with Diamond 3.0k (C1997-2014 Crystal Impact GbR, Bonn, Germany).

X-ray-quality crystals of the compound $\mathbf{2 f}$ (prismatic habit, colorless) were grown by slow evaporation ( $\sim 10 \mathrm{hrs}$ ) from a 1:1 mixture of hexane and $\mathrm{CH}_{2} \mathrm{Cl}_{2}$ at room temperature. The specimen used for the $\mathrm{X}$ ray diffraction experiment was cut from a larger agglomerate and polished by mechanical ablation in a drop of perfluorinated oil. It showed pleochroism under polarized light (from colourless to dark grey). 
$2 \mathbf{f}$ is chiral and crystallizes in the monoclinic centric space group $\mathrm{P} 2{ }_{1} / \mathrm{n}$ as a racemate, with one molecule per asymmetric unit and absolute configurations $(S, S)$ or $(R, R)$. The oxazolidin-2-one ring is slightly distorted toward a half-chair conformation. Unit cell $\left(\AA, \operatorname{deg}, \AA^{3}\right)$, as estimated from 9987 intense reflections with $4.7 \leq 2 \theta \leq 56.2 \mathrm{deg}: a=7.8801(2), b=17.1890(5), c=11.5884(3), \beta=96.156(1), V=$ 1560.6(1).

X-ray quality crystals (prismatic habit, colorless) were grown by slow evaporation ( $~ 6 \mathrm{hrs})$ from a 1:1 hexane: $\mathrm{CH}_{2} \mathrm{Cl}_{2}$ mixture at room temperature. The sample chosen for the $\mathrm{X}$-ray analysis was cut from a larger agglomerate and polished by mechanical ablation in a drop of perfluorinated oil. It showed pleochroism under polarized light (from colourless to dark grey). The compound is chiral and crystallizes in the orthorhombic acentric space group P $2{ }_{1} 2_{1} 2_{1}$ as a pure enantiomer, with one molecule per asymmetric unit. The presence of sulphur and iodine anomalous scatterers allow to secure the absolute molecular configuration, with a Flack parameter ${ }^{[53]}$ as low as $0.02(2)$ by classical fit to all intensities (Figure 3). The saturated six-membered ring assumes an almost perfect chair conformation, while the oxazolidin-2-one ring, analogously to compound $\mathbf{2 f}$, adopts a slightly distorted half-chair conformation. Unit cell $\left(\AA, \AA^{3}\right)$, as estimated from 8060 intense reflections with $5.3 \leq 2 \theta \leq 47.2$ deg: $a$ $=8.1577(2), b=11.8874(2), c=20.0540(4), V=1944.7(1)$.

\section{Acknowledgements}

Università degli Studi dell'Insubria and Università degli Studi di Milano are acknowledged for financial support.

\section{Author Contribution Statement}

G. B. and E. M. B. designed the project. S. G. and R. S. performed the experiments. XRD data was acquired and analyzed by L. L. P. All authors contributed to writing the manuscript.

\section{References}

[1] S. R. Chemler, M. T. Bovino, 'Catalytic Aminohalogenation of Alkenes and Alkynes', ACS Catal. 2013, 3, 1076-1091.

[2] C. K. Tan, Y. Y. Yeung, 'Recent Advances in Stereoselective Bromofunctionalization of Alkenes using N-Bromoamide Reagents', Chem. Commun. 2013, 49, 7985-7996.

[3] E. M. G. Broggini, T. Borelli, S. Giofré A. Mazza, 'Intramolecular Oxidative Palladium-Catalyzed Amination Involving Double C-H Functionalization of Unactivated Olefins', Synthesis 2017, 49, 2803- 2818.

[4] G. Q. Liu, W. Li, Y. M. Li, 'A New Method for Intramolecular Chloroamination of Unfunctionalized Olefins', Adv. Synth. Catal. 2013, 355, 395-402. 
[5] M. R. Manzoni, T. P. Zabawa, D. Khasi, S. R. Chemler, 'Palladium(II)-Catalyzed Intramolecular Aminobromination and Aminochlorination of Olefins', Organometallics 2004, 23, 5618-5621.

[6] D. H. Horning, J. M. Muchowski, 'Intramolecular Bromination. Part I', Can. J. Chem. 1974, 52, $1321-1330$.

[7] W. Li, G.-Q. Liu, B. Cui, L. Zhang, T.-T. Li, L. Li, L. Duan, Y.-M. Li 'Transition Metal-Free Iodine-Promoted Haloamination of Unfunctionalized Olefins', RSC Adv. 2014, 4, 13509-13513.

[8] J. Qiu, R. B. Silverman, 'A New Class of Conformationally Rigid Analogues of 4-Amino-5halopentanoic Acids, Potent Inactivators of $\gamma$-Aminobutyric Acid Aminotransferase', J. Med. Chem. 2000, 43, 706-720.

[9] K. Pors, S. D. Shnyder, P. H. Teesdale-Spittle, J. A. Hartley, M. Zloh, M. Searcey, L. H. Patterson, 'Synthesis of DNA-Directed Pyrrolidinyl and Piperidinyl Confined Alkylating Chloroalkylaminoanthraquinones: Potential for Development of Tumor-Selective $N$-Oxides', $J$. Med. Chem. 2006, 49, 7013-7023.

[10]A. F. French, S. Bissmire, T. Wirth, 'Iodine Electrophiles in Stereoselective Reactions: Recent Developments and Synthetic Applications', Chem. Soc. Rev. 2004, 33, 354-362.

[11]P. Mizar, T. Wirth, 'Iodoaminations of Alkenes', Synthesis 2017, 49, 981-986.

[12] O. Kitagawa, N. Kikuchi, T. Hanano, K. Aoki, T. Yamazaki, M. Okada, T. Taguchi, 'A Mild and Highly Chemoselective $\alpha$-Iodination of $N$-Allylic Carboxamides and Lactams', J. Org. Chem. 1995, 60, 7161-7165.

[13]M. Fujita, O. Kitagawa, T. Suzuki, T. Taguchi, 'Regiocontrolled Iodoaminocyclization Reaction of an Ambident Nucleophile Mediated by Basic Metallic Reagent', J. Org. Chem. 1997, 62, 73307335.

[14] O. Kitagawa, T. Suzuki, T. Taguchi, ' $t$-BuOK-Mediated Iodoaziridination Reaction of N-Allylic Tosylamide Derivatives', J. Org. Chem. 1998, 63, 4842-4845.

[15]Y. Wang, K.-F. Wang, Y. Su, Z. Yang, L. Wen, L. Liu, J. Wang, D. Huang, Y. Hu, 'Cascade Oxidation/Halogenoaminocyclization Reaction of Trifluoromethylated Homoallylic $\mathrm{N}$ Acylhydrazines: Metal-free Synthesis of $\mathrm{CF}_{3}$-Substituted Pyrazolines', J. Org. Chem. 2018, 83, 939-950.

[16]S. Knapp, K. E. Rodriques, A. T. Levorse, R. M. Omaf, “A Procedure for "Iodolactamization”", Tetrahedron Lett. 1985, 26, 1803-1806.

[17] A. Bongini, G. Cardillo, M. Orena, S. Sandri, C. Tomasini, 'Factors Affecting the Regioselection of Allylic Imidate Iodocyclization', J. Org. Chem. 1986, 51, 4905-4910.

[18] C. S. Brindle, C. S. Yeung, E. N. Jacobsen, 'Chiral $\beta$-Iodoamines by Urea-Catalysed Iodocyclization of Trichloroacetimidates', Chem. Sci. 2013, 4, 2100-2104. 
[19]G. Q. Liu, Y. M. Li, 'Regioselective (Diacetoxyiodo)benzene-Promoted Halocyclization of Unfunctionalized Olefins', J. Org. Chem. 2014, 79, 10094-10109.

[20] M. T. Bovino, S. R. Chemler, 'Catalytic Enantioselective Alkene Aminohalogenation/Cyclization Involving Atom Transfer', Angew. Chem., Int. Ed. 2012, 51, 3923-3927.

[21]L. Chen, X. Luo, Y. Li, 'Palladium-Catalyzed Intramolecular Aminoiodination of Alkenes Using Molecular Oxygen as Oxidant', Monatsh. Chem. 2017, 148, 957-961.

[22] H. Sun, B. Cui, G.-Q. Liu, Y.-M. Li, ' $\mathrm{MnI}_{2}$-Catalysed Regioselective Intramolecular Iodoamination of Unfunctionalized Olefins', Tetrahedron 2016, 72, 7170-7178.

[23]H. Sun, B. Cui, L. Duan, Y.-M. Li, 'Intramolecular Aminoalkoxylation of Unfunctionalized Olefins via Intramolecular Iodoamination and Aziridinium Ion Ring-Opening Sequence', Org. Lett. 2017, 19, 1520-1523.

[24]H. Liu, Y. Pan, C. Tan, 'Sodium Nitrite $\left(\mathrm{NaNO}_{2}\right)$ Catalysed Iodo-cyclisation of Alkenes and Alkynes using Molecular Oxygen’, Tetrahedron Lett. 2008, 49, 4424-4426.

[25] M. Hashimoto, M. Matsumoto, K. Yamada, S. Terashima, 'Synthetic Studies of Carzinophilin. Part 4: Chemical and Biological Properties of Carzinophilin Analogues', Tetrahedron 2003, 59, 30893097.

[26]I. Pérez-Castro, O. Caamaňo, F. Fernàndez, M.D. Garcìa, C. Lopez, E. De Clercq, 'Synthesis of 4Substituted-1,2,3-triazole Carbanucleoside Analogues of Ribavirin via Click chemistry', Org. Biomol. Chem. 2007, 5, 3805-3813.

[27] S. Gazzola, E. M. Beccalli, T. Borelli, C. Castellano, D. Diamante, G. Broggini, 'Selective 7-endoCyclization of 3-Aza-5-alkenols through Oxidative Pd(II)-Catalyzed Olefin Oxyarylation', Synlett 2018, 29, 503-508.

[28] G. Broggini, E. M. Beccalli, T. Borelli, F. Brusa, S. Gazzola, A. Mazza, 'Intra-Intermolecular Palladium-Catalyzed Domino Reactions of Glycine Allylamides for the Synthesis of Diversely Functionalized Piperazinones', Eur. J. Org. Chem. 2015, 4261-4268.

[29] G. Broggini, V. Barbera, E. M. Beccalli, U. Chiacchio, A. Fasana, S. Galli, S. Gazzola, 'Selective Intramolecular Palladium(II)-Catalyzed Aminooxygenation vs. Diamination of Alkenylureas: Efficient Microwave-Assisted Reactions to Bicyclic Piperazinones', Adv. Synth. Catal. 2013, 355, 1640-1648.

[30]E. Borsini, G. Broggini, A. Fasana, S. Galli, M. Khansaa, U. Piarulli, M. Rigamonti, 'Intramolecular Palladium-Catalyzed Aminocarboxylation of Olefins as a Direct Route to Bicyclic Oxazolidinones', Adv. Synth. Catal. 2011, 353, 985-994.

[31] T. Froehr, C. P. Sindlinger, U. Kloeckner, P. Finkbeiner, B. J. Nachtsheim, 'Benzoxazoles The First Example of an Iodide-Catalyzed Oxidative Amination of Heteroarenes', Org. Lett. 2011, 13, 3754-3757. 
[32] A. Fürst, P. A. Plattner, ' $2 \alpha, 3 \alpha$ - und $2 \beta, 3 \beta$-Oxido-chlolestane; Konfiguration der 2-Oxycholestane' Helv. Chim. Acta 1949, 32, 275-283.

[33] G. Bellucci, G. Berti, G. Ingrosso, A. Vatteroni, G. Conti, R. Ambrosetti, 'Influence of Substituents in the 3-Position of Cyclohexene Oxides on the Rates of their Reactions with Hydrogen Chloride in Low Polarity Aprotic Solvents', J. Chem. Soc., Perkin Trans. 2 1978, 627-632.

[34] G. Li, L. Huang, J. Xu, W. Sun, J. Xie, L. Hong, R. Wang, 'Sodium Iodide/Hydrogen PeroxideMediated Oxidation/Lactonization for the Construction of Spirocyclic Oxindole-Lactones', Adv. Synth. Catal. 2016, 358, 2873-2877.

[35]A. Yoshimura, C. Zhu, K. R. Middleton, A. D. Todora, B. J. Kastern, A. V. Maskaev, V. V. Zhdankin, 'Hypoiodite Mediated Synthesis of Isoxazolines from Aldoximes and Alkenes Using Catalytic KI and Oxone as the Terminal Oxidant', Chem. Commun. 2013 49, 4800-4802.

[36] V. K. Yadav, V. P. Srivastava, L. D. S. Yadav, ' Iodide Catalyzed Synthesis of 2Aminobenzoxazoles via Oxidative Cyclodesulfurization of Phenolic Thioureas with Hydrogen Peroxide', Tetrahedron Lett. 2018, 59, 252-255.

[37] M. Uyanik, H. Okamoto, T. Yasui, K. Ishihara, 'Quaternary Ammonium (Hypo)iodite Catalysis for Enantioselective Oxidative Cycloetherification', Science 2010, 328, 1376-1379.

[38]C. Fortin, S. Khanniche, D. Khiri, V. Fèvre-Nollet, P. Lebègue, F. Cousin, I. Černušàk, F. Louis, 'Reactivity of Hydrogen Peroxide with Br and I Atoms', J. Phys. Chem. 2018, 122, 1053-1063.

[39]T. K. Achar, S. Maiti, P. Mal, 'PIDA-I2 Mediated Direct Vicinal Difunctionalization of Olefins: Iodoazidazion, iodoetherification and iodoacyloxylation', Org. Biomol. Chem. 2016, 14, 4654.

[40]K. B. Hong, J. N. Johnston, 'Alkene Diamination Using Electron-Rich Amines: Hypervalent Iodine-Promoted Inter/Intramolecular C-N Bond Formation', Org. Lett. 2014, 16, 3804-3807.

[41]Y.-T. Gao, X.-Y. Jin, Q. Liu, A.-D. Liu, L. Cheng, D. Wang, 'Iodide/H2O2 Catalyzed Intramolecular Oxidative Amination for the Synthesis of 3,2'-Pyrrolidinyl Spirooxindoles', Molecules 2018, 23, 2265.

[42] S. Gazzola, E. M. Beccalli, T. Borelli, C. Castellano, M. A. Chiacchio, D. Diamante, G. Broggini, 'Copper(II)-Catalyzed Alkoxyhalogenation of Alkynyl Ureas and Amides as a Route to Haloalkylidene-Substituted Heterocycles', J. Org. Chem. 2015, 80, 7226-7234.

[43]K. Muniz, A. Iglesias, Y. Fang, 'Platinum-Catalysed Aerobic 1,2-Aminooxygenation of Alkenes', Chem. Commun. 2009, 5591-5593.

[44] A. Gimeno, A. B. Cuenca, M. Médio-Simon, G. Asensio, 'Gold(I)-Catalyzed Reactions of 1-(orthoAlkynylaryl)ureas: Highly Selective Heterocyclization and Synthesis of Mixed $N, O$-Acetals', $A d v$. Synth, Catal. 2014, 356, 229-236. 
[45]E. M. Beccalli, G. Broggini, G. Paladino, A. Penoni, C. Zoni, Regioselective Formation of Six- and Seven-Membered Ring by Intramolecular Pd-Catalyzed Amination of $N$-Allyl-anthranilamides, $J$. Org. Chem. 2004, 69, 5627-5630.

[46] A. D. Manick, F. Berhal, G. Prestat, 'Synthesis of Six- and Seven-Membered ChloromethylSubstituted Heterocycles via Palladium-Catalyzed Amino- and Oxychlorination', Synthesis 2016, 48, 3719-3729.

[47] G. Abbiati, E. M. Beccalli, G. Broggini, M. Martinelli, G. Paladino, 'Pd-Catalyzed Cyclization of 1-Allyl-2-indolecarboxamides by Intramolecular Amidation of Unactivated Ethylenic Bond', Synlett 2006, 73-76.

[48] Bruker AXS Inc., SAINT+, Madison, Wisconsin, USA, 2012.

[49] Bruker AXS Inc., SADABS, Madison, Wisconsin, USA, 2001.

[50]L. Palatinus, G. Chapuis, 'SUPERFLIP - A Computer Program for the Solution of Crystal Structures by Charge Flipping in Arbitrary Dimensions', J. Appl. Crystallogr. 2007, 40, 786-790.

[51]A. Altomare, G. Cascarano, C. Giacovazzo, A. Guagliardi, 'Completion and Refinement of Crystal Structures with SIR92', J. Appl. Crystallogr. 1993, 26, 343-350.

[52] G. M. Sheldrick, 'SHELXT - Integrated Space-group and Crystal-structure Determination', Acta Crystallogr. 2015, 71, 3-8.

[53]H. D. Flack., 'On Enantiomorph-polarity Estimation', Acta Crystallogr. 1983, A39, 876-881. 